\title{
Range Estimation of a Moving Target Using Ultrasound Differential Zadoff-Chu Codes
}

\author{
Mohammed H. AlSharif ${ }^{(0)}$, Student Member, IEEE, Mohamed Saad ${ }^{\circledR}$, Member, IEEE, \\ Mohamed Siala ${ }^{\circledR}$, Member, IEEE, Mohanad Ahmed ${ }^{\circledR}$, Member, IEEE, \\ and Tareq Y. Al-Naffouri ${ }^{\circledR}$, Senior Member, IEEE
}

\begin{abstract}
High-accuracy range estimation is essential in modern applications and technologies. However, it is challenging to estimate the continuous-range of a moving target, especially under Doppler effects. This article presents a novel signal design, which we name differential Zadoff-Chu (DZC). Under Doppler effects, DZC sequences improve the performance of the maximum likelihood (ML)-based range estimation over its performance when using regular Zadoff-Chu (ZC) sequences. We propose a reduced-complexity ranging algorithm using DZC sequences and show that it outperforms the regular $\mathrm{ZC}$ ML-based range estimation. We evaluate the proposed system in a typical indoor environment using a low-cost ultrasound hardware. Under a low signal-to-noise ratio ( $-10 \mathrm{~dB}$ SNR), more than $90 \%$ of the range estimates have less than a 1.6-mm error, with a movement range from 0.2 to $2.2 \mathrm{~m}$ and a maximum velocity of $0.5 \mathrm{~m} / \mathrm{s}$. For the same movement range, the system provides range estimates with a root-mean-square error (RMSE) of less than $0.76 \mathrm{~mm}$ in a high SNR scenario (10 $\mathrm{dB})$, and an RMSE less than $0.85 \mathrm{~mm}$ in a low SNR scenario $(-10 \mathrm{~dB})$. For a larger movement range from 1.8 to $4.2 \mathrm{~m}$ with a maximum velocity of $1.91 \mathrm{~m} / \mathrm{s}$, the proposed system provides range estimates with an RMSE less than $7.70 \mathrm{~mm}$ at $10 \mathrm{~dB}$ SNR.
\end{abstract}

Index Terms-Differential coding, differential Zadoff-Chu (DZC) sequences, Doppler estimation, maximum-likelihood (ML) estimation, movement estimation, ultrasound.

\section{INTRODUCTION}

$\mathbf{M}$ ANY modern applications, including navigation, medical care, location-aware networks, video gaming, and virtual reality, require estimating the range between two devices with very high accuracy. Consequently, range estimation has been studied using varying approaches based on ultrasound, radio, infrared, and laser signals [1]-[4].

Although algorithms based on infrared and lasers have high accuracy, they are complicated and expensive [5]. Likewise, radio-signal-based range estimation approaches using the received signal strength (RSS) of a Wi-Fi or Bluetooth signal require precalibration and provide low accuracy [6]. While

Manuscript received November 16, 2020; revised March 3, 2021; accepted March 13, 2021. Date of publication April 12, 2021; date of current version April 26, 2021. This work was supported by the KAUST-MITTUD Consortium under Grant OSR-2015-Sensors-2700. The Associate Editor coordinating the review process was Alessio De Angelis. (Corresponding author: Mohammed H. AlSharif.)

Mohammed H. AlSharif, Mohanad Ahmed, and Tareq Y. Al-Naffouri are with the Division of Computer, Electrical and Mathematical Sciences and Engineering, King Abdullah University of Science and Technology, Thuwal 23955, Saudi Arabia (e-mail: mohammed.alsharif@kaust.edu.sa).

Mohamed Saad is with the Insight Research Centre for Data Analytics, Univeristy College Dublin, Dublin 4, D04 V1W8 Ireland.

Mohamed Siala is with the Department of Applied Mathematics, Signals and Communication, Higher School of Communication of Tunis (Sup'Com), Ariana 2083, Tunisia.

Digital Object Identifier 10.1109/TIM.2021.3072691 radio-based ranging methods, through time of flight (TOF) estimation, do not require precalibration [7], they still need accurate synchronization. In fact, due to the high speed of light, small timing errors result in large ranging errors. Therefore, approaches based on ultrawideband (UWB) radio signals typically have an accuracy level of 10-20 cm [8]. In contrast, ultrasound-based methods are of low cost and have high levels of accuracy in estimating the TOF of the signal, thanks to the low propagation speed of ultrasound signals [9]. Indeed, one of the widely used commercial UWB positioning systems is Pozyx [10] which claims a positioning accuracy of up to $10 \mathrm{~cm}$. On the other hand, the commercially available ultrasound-based positioning system, Marvel Mind [11], claims a positioning accuracy of $2 \mathrm{~cm}$. In this article, we focus on ultrasound-based ranging because of its greater accuracy.

Regardless of which technology is used, ranging waveforms can be categorized into two general classes: continuous wave $(\mathrm{CW})$ and pulsed. Despite being more susceptible to multipaths and echos, $\mathrm{CW}$ ranging still has many advantages over pulsed ranging; its main one being that it maximizes the total transmitted power, since the transmitter is continuously transmitting. Moreover, $\mathrm{CW}$ ranging can provide a much higher update rate, i.e., the number of range estimates per second as compared to pulsed ranging. Therefore, in this article, we focus on continuous-range estimation [12].

In addition to the aforementioned ranging technologies, the literature proposed various range estimation methods. Although RSS-based ranging algorithms are generally simple compared to other algorithms, they suffer from low level of accuracy [13]. By contrast, phase shift-based ranging using a single frequency signal has high level of accuracy. However, this latter application is limited to distances of less than one wavelength of the signal carrier [14]. Using multiple frequencies allows the estimation of longer distances by calculating phase differences between the various frequencies. The authors in [15] use a narrowband multifrequency-continuouswave $(\mathrm{MFCW})$ ultrasound signal for range estimation. In the MFCW, the difference between the frequencies, $\Delta f$, restricts the estimated range to $c / \Delta f$ where $c$ is the speed of sound. Furthermore, range estimation based on narrowband signals is very sensitive to noise, multipath, and interference. To minimize the limitations of narrowband signals in TOF estimation, a number of range estimation systems using wideband signals have been proposed.

In basic TOF-based ranging systems, assuming that the transmitter and the receiver are synchronized, the peak location of the cross correlation between the transmitted 
and the received signals provides an estimate of the TOF. Indeed, perfect synchronization can be achieved through having the transmitter and the receiver share the same clock. After that, multiplying the TOF by the propagation speed of the signal results in the range between the transmitter and the receiver. Hence, the correlation properties of the transmitted signal strongly influence the accuracy of the estimated TOF. Due to their excellent correlation properties, Zadoff-Chu (ZC) sequences are widely used for synchronization purposes. In our previous work [16], a high accuracy TOF-based ranging system was implemented using a ZC-coded ultrasound signal. However, with a moving transmitter or receiver, Doppler shifts were found to severely degrade the accuracy of the range estimation, especially for high-speed moving devices and/or long transmitted signals.

We can estimate the range of a moving target by transmitting sine waves at multiple frequencies, followed by a frequency-modulated-continuous-wave (FMCW) [17]. The authors in [17] use the fast Fourier transform (FFT) of the sine waves and the FMCW to determine the velocity and range of the moving target, respectively. The downside of this approach is that the authors must assume a constant velocity over the duration of the transmitted signal, which limits its application to targets with low acceleration.

Under a low-acceleration assumption, a joint maximumlikelihood (ML) estimation of the target's velocity and range achieves very high accuracy [18]. However, as we will show, the ML approach requires a 2-D search which has high computational complexity [19]. Furthermore, if the target has a high acceleration, an additional search dimension is required to determine the target's acceleration.

Low-complexity solutions for removing the Doppler shift include differentially encoding the transmitted signal and differential decoding the received signal. In [20], the authors propose differential polyphase codes to resolve the frequency ambiguity. Another implementation of differential encoding and decoding is demonstrated in [21]. In this article, we propose a new signal design based on differential encoding.

The main contributions of this article are as follows:

1) We propose a novel signal design, which we name differential ZC, and we study its properties.

2) We derive the ML estimator and use it to estimate the TOF and Doppler shift.

3) Using differential Zadoff-Chu (DZC) sequences, we propose a low-complexity ranging algorithm and show that, under Doppler, it outperforms the benchmark ranging algorithms namely the regular ZC ML-based algorithm, the FMCW short-time-Fouriertransform (STFT)-based algorithm [17], multiple-signalclassification (MUSIC)-based line spectral estimation (LSE) [22], and the super-resolution radar, via $\ell_{1}$ minimization program [23].

4) We experimentally evaluate the proposed ranging system in a typical indoor environment using low-cost ultrasound hardware.

\section{PROBLEM Formulation}

ZC sequences are polyphase complex-valued sequences, named after Solomon A. Zadoff and D. C. Chu [24], [25].
These sequences have constant-amplitude-and-zero-autocorrelation (CAZAC) property, which allows them to provide a high-accuracy TOF-based range estimation of a static target, using cross correlation [16]. However, as we will show in this section, random Doppler shifts tend to break the CAZAC property of the ZC sequences, especially for long sequences and/or high-velocity moving targets.

Under Doppler, a wideband signal encounters time scaling (compression and/ or expansion) proportional to the relative speed between the transmitter and the receiver. Therefore, the passband-received ultrasound signal can be modeled as in [26]

$$
y(t)=\alpha(t) x((1+\Delta)(t-\kappa)) e^{i(\theta)}+n(t)
$$

where $x(t)$ is the passband-transmitted signal, $\alpha(t)$ is the attenuation incurred by propagation, $\Delta$ is the relative Doppler shift defined as the ratio of the relative velocity $v(t)$ to the speed of sound $c, \kappa$ is the TOF, $\theta$ is the overall phase shift encountered by the carrier, and $n(t)$ is an additive Gaussian noise, with zero mean and variance $\sigma^{2}$.

The time scaling of the received signal is the result of Doppler translating each frequency component by a different amount [26]. We propose two ranging algorithms in this article, one based on ML estimation and the other a low-complexity ranging algorithm. In ML-based ranging, we use the baseband version of the wideband-received-signal model given by (1). Whereas, in the low-complexity ranging algorithm, we approximate the received signal model using the narrowband time-delayed and Doppler shifted signal model [27]. To compensate for the error from the narrowband approximation, we apply a phase shift refinement algorithm using the wideband model (1). Therefore, the narrowband approximation of the received signal can be written as

$$
y(t)=\alpha(t) x(t-\kappa) e^{i(\theta+2 \pi v(t) t)}+n(t)
$$

where $v(t)$ is the Doppler shift at time $t$. The complex envelope, $y_{e}(t)$, of the received signal can be obtained using an IQ demodulator. Moreover, the discrete-time version of the received signal's complex envelope, obtained by sampling $y_{e}(t)$, at sampling period $T_{s}$, is given by

$$
\begin{aligned}
y_{e}[k] & =h[k] x_{e}[k-\tau]+n[k] \\
& =\alpha[k] x_{e}[k-\tau] e^{i(\theta+2 \pi \nu[k] k)}+n[k]
\end{aligned}
$$

where $h[k]=\alpha[k] e^{i 2 \pi v[k] k}$ is the channel response at discretetime $k$ under Doppler, $x_{e}[k]$ is the discrete-time complex envelope of the transmitted signal, $\tau$ is the TOF normalized by $T_{s}$ and rounded to the nearest integer, $v[k]$ is the discrete-time normalized Doppler shift, and $n[k]$ is a discretetime-complex-additive Gaussian noise with zero mean and variance $\sigma^{2}$. In addition, the transmitted signal is composed of repetitions of a sequence of length $N$, whose symbols we assume are transmitted according to the sampling rate $f_{s}=1 / T_{s}$. Therefore, the transmitted signal is periodic and this periodicity is required in our ranging algorithms, as will be illustrated in Section IV. If the target displacement over the sequence duration is less than several meters, then the change in the attenuation factor $\alpha[k]$ over the sequence 
duration is negligible [28]. In this article, this assumption is met. Therefore, it can be assumed that $\alpha[k]=\alpha_{B}$ for $k=0,1, \ldots, N-1$.

Let the complex envelope of the transmitted signal $x_{e}[k]$ be a ZC sequence of length $N$ given by

$$
x_{e}[k]=e^{i \phi_{R}[k]}
$$

where $\phi_{R}[k]$ is given by

$$
\phi_{R}[k]= \begin{cases}\frac{M \pi}{N} k(k+1), & \text { if } N \text { is odd } \\ \frac{M \pi k^{2}}{N}, & \text { if } N \text { is even }\end{cases}
$$

and $M, 0<M<N$ is coprime with $N$. It can be shown that in the noiseless case, and under a fixed (i.e., constant over the duration of the ZC sequence) Doppler shift $v$, the magnitude of the cross correlation $r[n]$ between the complex envelope of the transmitted and received signal is given by [16]

$$
\begin{aligned}
|r[n]| & \triangleq\left|\sum_{k=0}^{N-1} x_{e}^{*}[k] y_{e}[k+n]\right| \\
& =\left|\alpha_{B}\right|\left|\frac{e^{-i 2 \pi M\left(\tau-n-\frac{N v}{M}\right)}-1}{e^{-i 2 \pi \frac{M}{N}\left(\tau-n-\frac{N v}{M}\right)}-1}\right| \\
& =\left|\alpha_{B}\right|\left|\frac{\sin \left(\pi M\left(\tau-n-\frac{N v}{M}\right)\right)}{\sin \left(\pi \frac{M}{N}\left(\tau-n-\frac{N v}{M}\right)\right)}\right|, \quad n=0,1, \ldots, N-1 .
\end{aligned}
$$

This implies that the magnitude of the cross correlation function has a peak whenever both the numerator and the denominator are zeros. Therefore, the peak location depends on both the TOF $\tau$ and the Doppler shift $v$. Therefore, the location of this peak does not give the true TOF $\tau$, except when $v$ is zero. Actually, when $v$ is not zero, the location of the cross correlation peak is shifted in proportion to the value of $v$. As a result, the out-of-phase value of the crosscorrelation's magnitude is nonzero, i.e., the Doppler shift breaks the CAZAC property of the $\mathrm{ZC}$ sequences.

\section{DZC SEQuences AND Their Properties}

To mitigate Doppler shifts and to improve range estimation accuracy, we propose a new code $a[k]$, called DZC, given by

$$
a[k]=e^{i \phi_{D}[k]}, \quad k=0,1, \ldots, N-1
$$

where

$$
\phi_{D}[k]= \begin{cases}\frac{\pi M}{3 N} k(k+1)(k-1), & \text { for odd } N \\ \frac{\pi M}{3 N} k\left(k-\frac{1}{2}\right)(k-1), & \text { for even } N\end{cases}
$$

and $M, 0<M<N$, is coprime with $N$. The proposed sequences are named DZC sequences because they are derived based on differential sliding correlation defined in Section III-A. As we show in Appendix B, DZC sequences are periodic, with period $N$ if $N$ is odd and not divisible by 3 , $3 N$ if $N$ is odd and divisible by $3,4 N$ if $N$ is even and either $(2 N-1)$ or $(N-1)$ is divisible by 3 , and $12 N$ otherwise.

We claim that under the assumption of a high symbol rate, i.e., the number of symbols per second, DZC sequences almost preserve the CAZAC property, even in the presence of random Doppler shifts. Moreover, we claim that for any sequence length, $N$, using coherent detection, DZC sequences provide better range estimation accuracy compared to regular $\mathrm{ZC}$ sequences.

To prove the first claim, we utilize a differential-slidingcorrelation approach and show that, under a high symbol rate, DZC sequences almost preserve the CAZAC property, even if there are random Doppler shifts. To prove the second claim, we evaluate the root-mean-square-error (RMSE) for estimating the range and velocity of a moving target in regular and DZC sequences, both through simulation and through real experiments.

\section{A. Robustness to Doppler}

In this subsection, using the following differential-slidingcorrelation, we will show that DZC codes maintain the CAZAC property even under random Doppler shifts. The correlation is defined as

$$
r_{D}[n, m]=\sum_{k=0}^{N-1} x_{e}^{*}[k] x_{e}[k+m] y_{e}[k+n] y_{e}^{*}[k+m+n]
$$

for $n=0,1, \ldots, N-1$, where $m$ is the differentialcorrelation step. DZC sequences are designed such that for any differential-correlation step $m$, the product $x_{e}^{*}[k] x_{e}[k+m]$ is a regular ZC sequence. Therefore, we end up correlating the differentially received samples with a regular ZC sequence. Here we are applying circular differential-sliding-correlation in which $y_{e}[k+n+N] y_{e}^{*}[k+m+n+N]=y_{e}[k+n] y_{e}^{*}[k+m+n]$. The complex envelope of the received signal is cut into frames, each of length $N$. The differential decoded frame at instant $k$ is obtained by multiplying the frame at instant $k$ with the conjugate of the frame starting at $k+m$.

Now, consider an odd-length DZC. In the noiseless case and with $m=1$, using (3) and (8), we can rewrite the equation above as

$$
\begin{aligned}
r_{D} & {[n, m=1] } \\
& =\sum_{k=0}^{N-1} h^{*}[k] h[k+1] e^{-i \frac{\pi M}{N}(k-\tau+n)(k-\tau+n+1)} e^{i \frac{\pi M}{N} k(k+1)} .
\end{aligned}
$$

Under a high symbol rate with respect to the Doppler spread, it can be assumed that $h[k] \approx h[k+1]$, since for practical Doppler shifts, the channel will not change too much between two consecutive samples and hence $h^{*}[k]$ $h[k+1] \approx \alpha_{B}^{2}$, for any integer $k$. As an example, in our setup, the symbol duration is around $0.26 \mathrm{~ms}$. Therefore, even with an acceleration of $10 \mathrm{~m} / \mathrm{s}^{2}$, the change in velocity over one symbol duration will be $2.6 \mathrm{~mm} / \mathrm{s}$. This velocity change will shift a $20-\mathrm{kHz}$ signal by around $0.15 \mathrm{~Hz}$, which is negligible. Consequently, choosing $m=1$ removes the highest amount of Doppler residual.

Considering the noiseless case to simplify the analysis, the differential-sliding-correlation becomes

$r_{D}[n, m=1] \approx \sum_{k=0}^{N-1} \alpha_{B}^{2} e^{i \frac{\pi M}{N} k(k+1)} e^{-i \frac{i M}{N}(k-\tau+n)(k-\tau+n+1)}$ 


$$
\begin{aligned}
& =\alpha_{B}^{2} e^{-i \frac{\pi M}{N}(\tau-n)(\tau-n+1)} \sum_{k=0}^{N-1} e^{i \frac{\pi M}{N} 2 k(\tau-n)} \\
& =\alpha_{B}^{2} e^{-i \frac{\pi M}{N}(\tau-n)(\tau-n+1)} \frac{e^{i 2 \pi M(\tau-n)}-1}{e^{i 2 \pi \frac{M}{N}(\tau-n)}-1} .
\end{aligned}
$$

Taking the absolute value finally yields the desired result

$$
\left|r_{D}[n, m=1]\right|= \begin{cases}0, & \text { if } n \neq \tau \\ \left|\alpha_{B}\right|^{2} N, & \text { if } n=\tau .\end{cases}
$$

Similarly, we can show that an even-length DZC sequence has a differential-sliding-correlation also given by (13). This shows that the out-of-phase value for the magnitude of the differential-sliding-correlation is zero, hence the proposed DZC sequence maintains the CAZAC property, even under random Doppler shifts. Lastly, we would like to highlight that applying the differential sliding correlation to ZC sequences, with odd or even length $N$, will give a constant magnitude for all lags, i.e., $\left|r_{D}[n, m=1]\right|=N$ for all $n$. Therefore, applying differential correlation to $\mathrm{ZC}$ sequences does not provide a way to estimate the range of the target.

Finally, we would like to illustrate the capacity of the proposed DZC codes and differential correlation to estimate the ranges to multiple targets. To do so, each target transmits a unique DZC code of the same length (a DZC code with the same $N$ but different value of $M$ ). We address three scenarios in which we have three transmitters placed at three different distances; therefore, the received signal coming from each transmitter is delayed by a different TOF $\left(\tau_{1}=200, \tau_{2}=190\right.$, and $\tau_{3}=210$ ). In the first scenario, the three transmitters transmit the same DZC code. In Fig. 1(a), the received signal is composed of the same DZC code $\left(M_{1}=M_{2}=M_{3}=1\right)$, but with different time delays. We notice that the differential correlation gives three peaks located at the respective TOFs. This means that, since they have the same DZC code, the three transmitted signals are highly correlated, as expected. In the second scenario, each transmitter transmits a DZC code with the same $N$ but a different value for $M$. Fig. 1(b) shows the correlation between a DZC code with $M=1$ and the received signal, which is composed of three different DZC codes $\left(M_{1}=1, M_{2}=5\right.$, and $\left.M_{3}=9\right)$. Because of the orthogonality between the codes, the correlation vector has a single peak located at the TOF of the DZC code with $M_{1}=1$. In the last scenario, one transmitter transmits a DZC code and the other two transmitters transmit a ZC code. Fig. 1(c) shows the correlation vector between a DZC code with $M=1$ and the received signal, which is composed of a DZC code with $M_{1}=1$ and two ZC codes with $M_{2}=5$ and $M_{3}=9$. Again, because of the orthogonality between the codes, the correlation vector has a single peak located at the TOF of the first DZC code. Therefore, there is orthogonality between different DZC codes and between a DZC code and a $\mathrm{ZC}$ code. Consequently, by assigning each user a unique DZC code, we can perform multitarget range estimation.

\section{Range Estimation Algorithms}

In Section IV-A, we derive the ML estimator for both ZC and DZC sequences, which gives us a benchmark.

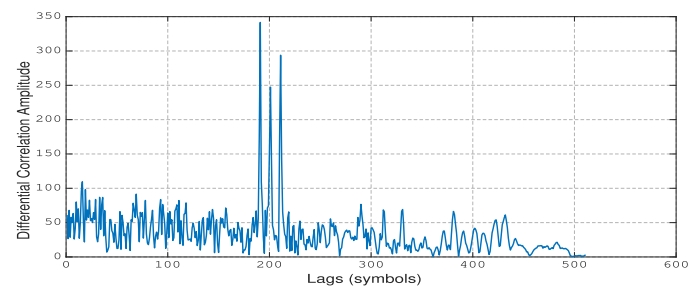

(a)

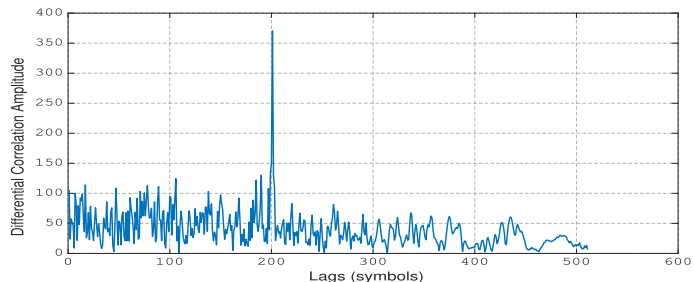

(b)

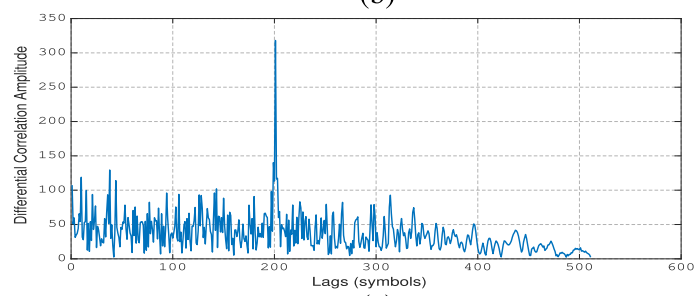

(c)

Fig. 1. Differential-correlation between a DZC code with $M=1$ and the received signal Rx composed of the addition of three codes delayed by $\tau_{1}=200, \tau_{2}=190$, and $\tau_{3}=210$. (a) Three DZC codes with $M_{1}=1$, $M_{2}=1$, and $M_{3}=1$. (b) Three DZC codes with $M_{1}=1, M_{2}=5$, and $M_{3}=9$. (c) One DZC code with $M_{1}=1$ and two ZC codes with $M_{2}=5$ and $M_{3}=9$.

In Section IV-B, we derive a low-complexity algorithm based on DZC sequences. While the performance of the lowcomplexity algorithm is inferior to the ML estimator applied to the DZC, this still outperforms the ML estimator applied to the ZC. Moreover, the low-complexity ranging algorithm accurately estimates the range even under random Doppler shifts, while the ML-based ranging algorithm requires a fixed Doppler shift over the duration of the transmitted sequence.

Finally, to compensate for the loss compared to the performance of the ML estimator, we improve the performance of the low-complexity method using a refinement algorithm based on phase shift estimation.

\section{A. ML Estimator}

Under a fixed Doppler shift over the duration of the transmitted sequence, the complex envelope of the received signal can be written as

$$
y_{e}(t)=x_{e}((1+\Delta)(t-\kappa)) e^{i(\theta+2 \pi v t)}+n(t)
$$

where $v=\left(f_{c} v / c\right)$ is the carrier frequency offset, $f_{c}$ is the carrier frequency of the transmitted signal, and $v$ is the velocity of the target over the duration of the transmitted signal. In this received signal model, the attenuation factor is assumed to be constant over the duration of the transmitted signal; hence, the attenuation factor appears as a scaling factor and can be easily estimated and removed. The discrete-time version of the signal in (14) is given by

$$
y_{e}[k]=x_{e}[(1+\Delta)(k-\tau)] e^{i(\theta+2 \pi v k)}+n[k] .
$$


The probability of the received sequence, whether regular or DZC, is given by

$$
\begin{aligned}
p(\mathbf{y} \mid \tau, \theta, v)= & \prod_{k=0}^{N-1} p\left(y_{e}[k] \mid \tau, \theta, v\right) \\
= & \frac{1}{\left(\pi \sigma^{2}\right)^{N}} \exp \left\{-\frac{1}{\sigma^{2}} \sum_{k=0}^{N-1} \mid y_{e}[k]\right. \\
& \left.-\left.x_{e}[(1+\Delta)(k-\tau)] e^{j(\theta+2 \pi v k)}\right|^{2}\right\} .
\end{aligned}
$$

The phase $\theta$ changes too much from one measurement to the other, so generally, precise a priori data on it can neither be obtained nor processed easily. The best approach is to assume the worst case scenario of a totally unknown phase with a uniform distribution over $[0,2 \pi)$. Averaging over $\theta$ enables us to focus on $\tau$ and $\nu$, on which conditioning remains after averaging. Therefore, the PDF in (16) becomes

$$
\begin{aligned}
p(\mathbf{y} \mid \tau, \nu)= & \int_{0}^{2 \pi} p(\mathbf{y} \mid \tau, \theta, \nu) p(\theta) d \theta \\
= & \int_{0}^{2 \pi} \frac{1}{2 \pi\left(\pi \sigma^{2}\right)^{N}} \exp \left\{\frac{-1}{\sigma^{2}} \sum_{k=0}^{N-1} \mid y_{e}[k]\right. \\
& \left.-\left.x_{e}[(1+\Delta)(k-\tau)] e^{j \theta} e^{j 2 \pi \nu k}\right|^{2}\right\} d \theta .
\end{aligned}
$$

By expanding the term inside the summation in (17), the likelihood function can be written as

$$
\begin{aligned}
p(\mathbf{y} \mid \tau, \nu) & \frac{1}{2 \pi\left(\pi \sigma^{2}\right)^{N}} \exp \left\{\frac{-1}{\sigma^{2}} \sum_{k=0}^{N-1}\left|y_{e}[k]\right|^{2}+\left|x_{e}[(1+\Delta)(k-\tau)]\right|^{2}\right\} \\
& \times \int_{0}^{2 \pi} \exp \left\{\frac{1}{\sigma^{2}} \sum_{k=0}^{N-1} 2 \Re\left\{y_{e}^{*}[k] x_{e}[(1+\Delta)(k-\tau)] e^{j \theta} e^{j 2 \pi v k}\right\} d \theta\right. \\
= & c_{\pi} e^{\eta(\tau)} \int_{0}^{2 \pi} \exp \left\{\frac{1}{\sigma^{2}}\left(\xi(\tau, v) e^{j \theta}+\xi^{*}(\tau, v) e^{-j \theta}\right)\right\} d \theta
\end{aligned}
$$

where

$$
\begin{aligned}
c_{\pi} & =\frac{1}{2 \pi\left(\pi \sigma^{2}\right)^{N}} \\
\xi(\tau, \nu) & =\sum_{k=0}^{N-1} y_{e}^{*}[k] x_{e}[(1+\Delta)(k-\tau)] e^{j 2 \pi v k} \\
\eta(\tau) & =-\frac{1}{\sigma^{2}} \sum_{k=0}^{N-1}\left(\left|y_{e}[k]\right|^{2}+\left|x_{e}[(1+\Delta)(k-\tau)]\right|^{2}\right) .
\end{aligned}
$$

Alternatively, we can write (18) as

$$
\begin{aligned}
& p(\mathbf{y} \mid \tau, v) \\
& =c_{\pi} e^{\eta(\tau)} \int_{0}^{2 \pi} \exp \left\{\frac{2}{\sigma^{2}}(|\xi(\tau, v)| \cos (\theta+L \xi(\tau, \nu))\} d . \theta\right. \\
& =c_{\pi} e^{\eta(\tau)} \int_{0}^{2 \pi} \exp \left\{\frac{2}{\sigma^{2}}(|\xi(\tau, v)| \cos (\theta)\} d \theta .\right. \\
& =2 \pi c_{\pi} e^{\eta(\tau)} I_{0}\left(\frac{2|\xi(\tau, v)|}{\sigma^{2}}\right)
\end{aligned}
$$

where (23) follows from the fact that the added angle $L \xi(\tau, v)$ has no effect on the integration of the cosine over its period. Here, $I_{0}($.$) is the modified Bessel function of the first kind and$ zero order. The modified Bessel function can be approximated as [29]

$$
I_{0}(z) \approx \frac{e^{z}}{\sqrt{2 \pi z}}, \text { for large } z
$$

Therefore, under a high SNR scenario, the likelihood function can be approximated as

$$
p(\mathbf{y} \mid \tau, \nu) \approx 2 \pi c_{\pi} e^{\eta(\tau)} \frac{\exp \left\{\frac{2|\xi(\tau, \nu)|}{\sigma^{2}}\right\}}{\sqrt{\frac{4 \pi|\xi(\tau, \nu)|}{\sigma^{2}}}} .
$$

Maximizing the likelihood function is equivalent to maximizing the log-likelihood function which, up to a constant, is given by

$$
\begin{aligned}
\ln p(\mathbf{y} \mid \tau, \nu) & \\
\approx & \frac{-1}{\sigma^{2}} \sum_{k=0}^{N-1}\left(\left|y_{e}[k]\right|^{2}+\left|x_{e}[(1+\Delta)(k-\tau)]\right|^{2}\right) \\
& +\frac{2}{\sigma^{2}}\left|\sum_{k=0}^{N-1} y_{e}^{*}[k] x_{e}[(1+\Delta)(k-\tau)] e^{j 2 \pi v k}\right| \\
& -\frac{1}{2} \ln \left(\frac{\left|\sum_{k=0}^{N-1} y_{e}^{*}[k] x_{e}[(1+\Delta)(k-\tau)] e^{j 2 \pi v k}\right|}{\sigma^{2}}\right)
\end{aligned}
$$

where we replace $c_{\pi}, \eta(\tau)$, and $\xi(\tau, v)$ by their expressions. In the high SNR scenario, the last term in (27) is negligible and the log-likelihood function reduces to

$$
\begin{aligned}
\ln p(\mathbf{y} \mid \tau, \nu) & \frac{-1}{\sigma^{2}} \sum_{k=0}^{N-1}\left(\left|y_{e}[k]\right|^{2}+\left|x_{e}[(1+\Delta)(k-\tau)]\right|^{2}\right) \\
& +\frac{2}{\sigma^{2}}\left|\sum_{k=0}^{N-1} y_{e}^{*}[k] x_{e}[(1+\Delta)(k-\tau)] e^{j 2 \pi \nu k}\right| .
\end{aligned}
$$

The first term can be ignored, since the signal itself has a constant amplitude and does not affect the maximization. Therefore, maximizing the log-likelihood function can be achieved by maximizing the following metric:

$$
\begin{aligned}
\left(\hat{\tau}_{\mathrm{ML}}, \hat{v}_{\mathrm{ML}}\right) & =\arg \max _{(\widetilde{\tau}, \widetilde{v})} M_{\tilde{\tau}, \widetilde{v}} \\
M_{\tilde{\tau}, \widetilde{v}} & =\left|\sum_{k=0}^{N-1} y_{e}[k] x_{e}^{*}[(1+\widetilde{\Delta})(k-\widetilde{\tau})] e^{-j 2 \pi \widetilde{v} k}\right|
\end{aligned}
$$

where $x_{e}[(1+\widetilde{\Delta})(k-\widetilde{\tau})]$ is obtained by resampling $x_{\tilde{\Delta}}[k-\widetilde{\tau}]$ to the new sampling frequency $f_{s}^{\prime}=(1+\widetilde{\Delta}) f_{s}$, with $\widetilde{\Delta}=\widetilde{v} / c$.

The argument of the module in (30) is known in pulsed radar and sonar signal processing as the ambiguity function, because it might have several maxima, which causes ambiguity in estimating $\tau$ and $\nu$. To illustrate this ambiguity, consider a transmitted odd-length ZC sequence and let us focus on the noiseless case with a negligible time scaling, i.e., $\Delta \ll 1$. Then, using (3) and (5), the metric (30) becomes

$$
\begin{aligned}
M_{\tilde{\tau}, \widetilde{v}} & =\left|e^{j \pi \frac{M}{N}\left(\tau^{2}+\tau-\widetilde{\tau}^{2}-\widetilde{\tau}\right)}\right|\left|\sum_{k=0}^{N-1} e^{j 2 \pi \frac{M}{N} k\left(\widetilde{\tau}-\tau+N \frac{v-\tilde{v}}{M}\right)}\right| \\
& =\left|\frac{e^{j 2 \pi M\left(\widetilde{\tau}-\tau+N \frac{\nu-\tilde{v}}{M}\right)}-1}{e^{j 2 \pi \frac{M}{N}\left(\widetilde{\tau}-\tau+N \frac{v-\tilde{v}}{M}\right)}-1}\right|
\end{aligned}
$$




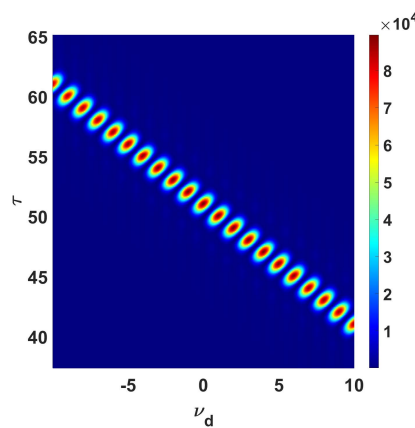

(a)

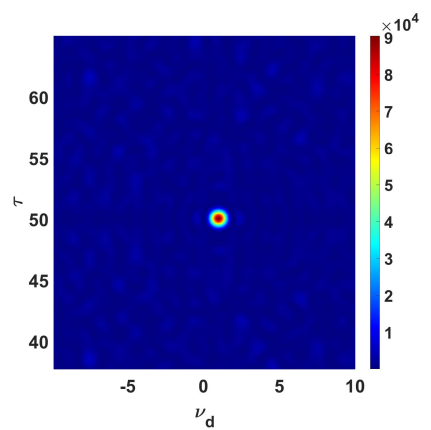

(b)
Fig. 2. Ambiguity function with $N=101, \tilde{\tau}=50$ symbols, $M=1$, and $\widetilde{v}=0.01$. (a) Regular ZC. (b) DZC.

where $\tau$ and $v$ are the true TOF and true Doppler shift, respectively. The metric in (31) has a maximum whenever the quantity $(\tilde{\tau}-\tau+N(v-\widetilde{v} / M))$ is an integer multiple of $N$, causing ambiguity in determining the TOF. Fig. 2(a) shows the ambiguity function with several maxima. To avoid this ambiguity, we limit our search over $\tau$ and $v$ to a window that includes a single maximum. Since the TOF hypothesis $\widetilde{\tau}$ is in $[0, N-1]$, the difference between the TOF hypothesis and the true TOF cannot exceed $N-1$. For this reason, we estimate the Doppler shift at time $i$ over a window centered at the previous Doppler shift estimate $\hat{v}_{i-1}$ and with a width equal to $M$, i.e., $\widetilde{v} \in\left(\hat{v}_{i-1}-(M / 2): v_{s}: \hat{v}_{i-1}+(M / 2)\right)$, where the search step $v_{s}$ is chosen to be small enough to detect the Doppler shift with high accuracy. For this estimation to be accurate, we need to know the initial Doppler shift $v_{0}$. Also, the current true Doppler shift $v_{i}$ should not differ from the previous Doppler shift $v_{i-1}$ by more than $(M / 2)$. Assuming that the target is initially static, makes the initial Doppler shift, $v_{0}$, equal to zero. To guarantee that the difference between the current and the previous Doppler shift is less than $(M / 2)$, we utilize the periodicity of $\mathrm{ZC}$ codes as will be shown in Section IV-B.

Unlike regular $\mathrm{ZC}$, using the proposed DZC sequences removes the ambiguity in estimating the TOF and Doppler shift. To illustrate this, consider an odd-length DZC sequence and let us focus again on the noiseless case. Then, using (3) and (8), the metric (30) becomes

$$
M_{\tilde{\tau}, \tilde{v}}=\left|\sum_{k=1}^{N} e^{j \pi \frac{M}{N} k\left((\tilde{\tau}-\tau) k+\left(\tau^{2}-\tilde{\tau}^{2}\right)+\frac{2 N(v-\widetilde{v})}{M}\right)}\right| .
$$

This metric has maxima at $\tau=\widetilde{\tau}$ and $(v-\widetilde{v})$ is an integer. Therefore, the ambiguity is removed over $\tau$, and there is no need to assume a known initial Doppler shift to unambiguously estimate the TOF. Fig. 2 shows the ambiguity functions for the regular $\mathrm{ZC}$ and $\mathrm{DZC}$ sequences.

\section{B. Reduced Complexity Ranging Algorithm}

The ML estimator has the lowest variance for all possible values of the estimation parameters but is computationally expensive. Therefore, we propose a reduced-complexity range estimation algorithm that makes use of the CAZAC property using the proposed DZC sequences.

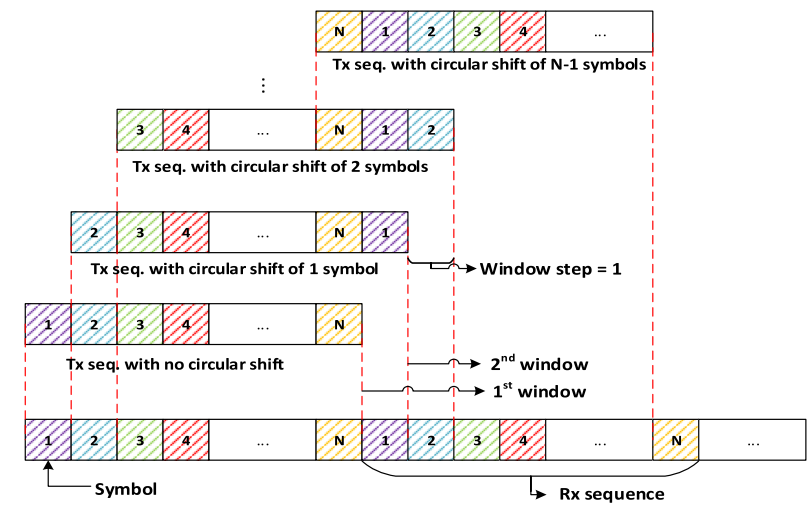

Fig. 3. Processing the received sequences.

For repetitive and periodic transmissions, the DZC sequence is repeated $P$ times. The received signal is processed using a sliding window with width $N$ to estimate the TOF and the Doppler shift. The step $w_{s}$ by which we move the window determines the system's update rate, $f_{u}$, or the number of estimates per second. Setting $w_{s}=1$ gives the highest possible update rate. Fig. 3 illustrates the processing of the received sequences, in which the sliding window is applied with a step of one symbol.

Consider the $(i+1)$ th window of the received signal's complex envelope, applying differential-sliding-correlation gives

$$
\begin{aligned}
r_{D}[n, m]=\sum_{k=0}^{N-1} x_{e}^{*}[k]_{i} x_{e}[k+ & m]_{i} y_{e}[k+n+i] \\
& \times y_{e}^{*}[k+m+n+i]
\end{aligned}
$$

where $x_{e}[k]_{i}$ is a circularly shifted version of the transmitted DZC sequence with phase function $\phi_{D}[k]_{i}=\phi_{D}[k+$ $i(\bmod N)], y_{e}[k]$ is the received sequence, and $N$ is the period of the sequence. The index $i+1$, where $i \in[0, p n-1]$ is the index of the range estimate, and $p n$ is the total number of range estimates. Therefore, with the circular-shift differentialsliding-correlation, each DZC sequence of length $N$ gives us $N$ TOF estimates. As was shown in (9)-(12), taking the absolute value of the differential-sliding-correlation gives

$$
\left|r_{D}[n, 1]\right|= \begin{cases}0, & \text { if } n \neq \tau \\ \left|\alpha_{B}\right|^{2} N, & \text { if } n=\tau .\end{cases}
$$

Therefore, the location of the maximum of $\left|r_{D}[n]\right|$ gives $\hat{\tau}_{\text {corr }}$, which we call the initial TOF estimate. Multiplying the initial TOF estimate by the speed of sound gives the initial range estimate $\hat{d}_{\text {corr }}$. The initial range estimates are accurate up to a sample resolution. If the true range is not an integer multiple of the sample resolution, then the fractional range will be rounded to the nearest sample. However, this rounding process causes errors in estimating the range; therefore, to achieve subsample resolution and to improve the ranging immunity to noise, we estimate the phase shift between the transmitted and the received DZC sequence and use it to refine the initial range estimates. To do that we need first to estimate and compensate for the Doppler shift. 


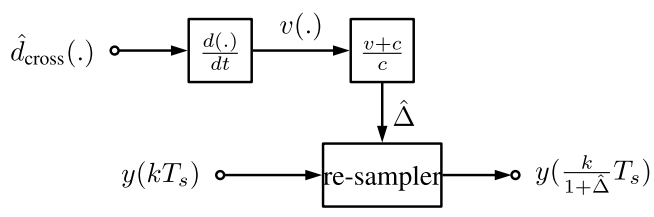

Fig. 4. Frequency offset estimation and compensation method.

1) Doppler Estimation and Compensation: To estimate the Doppler shift, we need to estimate the velocity over segments of the received sequence. The segment length $N_{s}$, which is a factor of the sequence length $N$, is chosen such that the change in velocity is negligible over the duration of the segment. The number of range estimates per segment is given by $L=\left(N / N_{s}\right)$. Differentiating these $L$ range estimates gives $L$ instantaneous velocity estimates per segment. Averaging the velocity estimates and dividing by the speed of sound $c$ gives the relative Doppler shift $\hat{\Delta}$ of that segment.

The frequency offset, caused by the Doppler shift, translates into a time scaling (compression or expansion) of the signal waveform [26], such that

$$
y\left(k T_{s}\right)=x\left(k(1+\Delta) T_{s}\right)
$$

where $x(t)$ and $y(t)$ are the transmitted and the received signal, respectively. Inverse-time-scaling the received signal using the estimated relative Doppler shift $\hat{\Delta}$ compresses or expands the signal and therefore removes the frequency offset. This is equivalent to resampling the bandpass signal by $1+\hat{\Delta}$, leading to

$$
x\left(k T_{s}\right)=y\left(\left(\frac{k}{1+\hat{\Delta}}\right) T_{s}\right) .
$$

The frequency-offset estimation-and-compensation algorithm is implemented in two steps, as shown in Fig. 4. First, we estimate the relative Doppler shift $\Delta$ using the initial range estimates. Then we resample the segments of the received signal to the new sampling frequency $f_{s}^{\prime}=(1+\hat{\Delta}) f_{s}$. Fig. 5 shows the transmitted and the received signals' spectra before and after frequency-offset compensation.

2) Phase Shift Estimation: After correcting the frequency offset of the received signal, we can refine the initial range estimates $\hat{d}_{\text {corr }}$ to a subsample resolution by estimating the phase shift between the transmitted and the corrected received signals.

Let the discrete-time version of the received signal after Doppler compensation be

$$
y[k]=x\left[k-\left\lfloor\frac{d}{c}\right\rceil \|\right]+n[k]
$$

where $[.7 \|$ denotes the operation of rounding to the nearest integer. The circularly shifted version of the transmitted signal, with a circular shift of $\left\lfloor\left(\hat{d}_{\text {corr }} / c\right)\right\rceil \|$, is given by

$$
z[k]=x\left[k-\left\lfloor\frac{\hat{d}_{\text {corr }}}{c}\right\rceil \|\right] .
$$

The phase shift between $y[k]$ and $z[k]$ at the $m$ th frequency bin $\omega_{m}$ is given by [30]

$$
\begin{aligned}
\hat{\phi}_{m} & =\operatorname{ang}\left(Z\left(\omega_{m}\right) Y^{*}\left(\omega_{m}\right)\right) \\
& =\omega_{m} \tau_{\phi}+\epsilon_{m}
\end{aligned}
$$

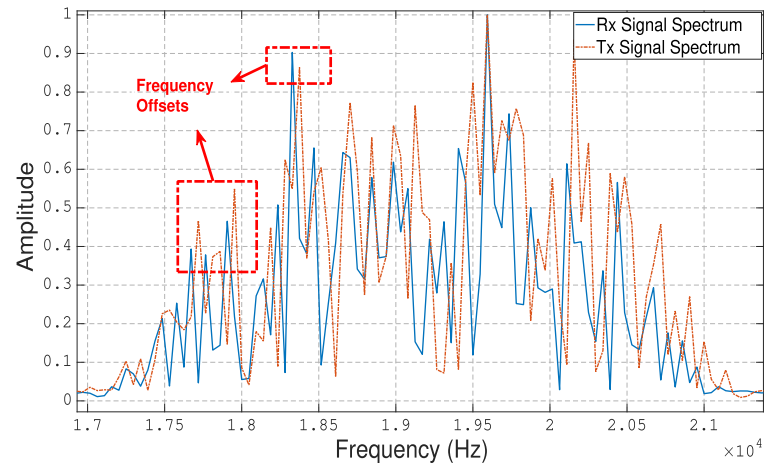

(a)

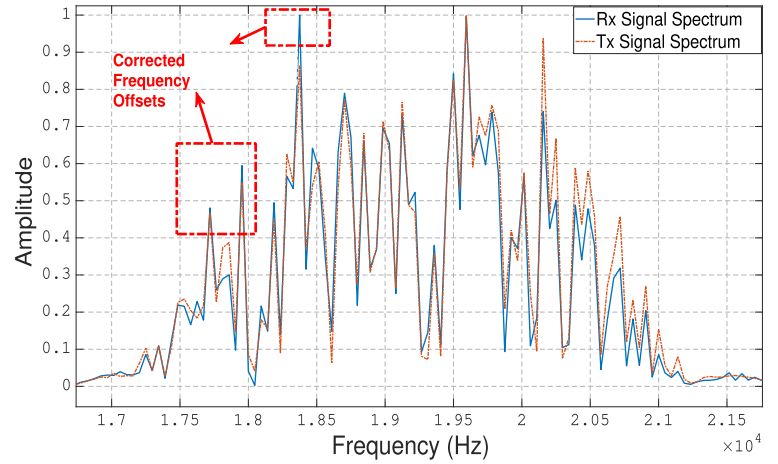

(b)

Fig. 5. Transmitted and the received signals spectra (a) before and (b) after frequency-offset compensation.

where $Z(\omega)$ and $Y(\omega)$ are the discrete Fourier transforms of $z[k]$ and $y[k]$, respectively, $*$ denotes the complex conjugation operation, $\tau_{\phi}$ is the subsample delay, and $\epsilon_{m}$ is the error in the estimated phase generated by noise. Dividing the estimated phase shift $\hat{\phi}_{m}$ by the associated frequency bin $\omega_{m}$ and multiplying it by the speed of sound $c$ gives the estimated range refinement $\hat{\Delta} d_{m}$.

Since the DZC sequences have frequencies that vary with time, it is necessary to estimate the phase shift associated with each of these frequencies. Therefore, we determine the valid frequency bins for the received signal based on a particular threshold value. This value is decided experimentally to be 0.5 of the maximum of $Z(\omega)$. All frequency bins that have components higher than this threshold are considered valid frequencies. The estimated range refinement $\hat{\Delta} d$ is the average of $\hat{\Delta} d_{m}$ for all valid frequency bins

$$
\hat{\Delta} d=\frac{\Sigma_{m=0}^{M-1} \hat{\Delta} d_{m}}{M}
$$

where $M$ is the number of the valid frequency bins. Finally, the refined range is given by

$$
\hat{d}=\hat{d}_{\mathrm{corr}}+\hat{\Delta} d .
$$

3) Minimum Refinement-Variance Search: Since the phase information is limited to $\pm \pi$, the range refinement $\Delta d$ is confined to one wavelength of the transmitted signal. Therefore, the phase shift algorithm fails when the absolute value of the error is larger than $\lambda_{\max } / 2$, where $\lambda_{\max }$ is the maximum wavelength of the signal. This error might be reached in low SNR conditions. To correct the initial range estimates to 


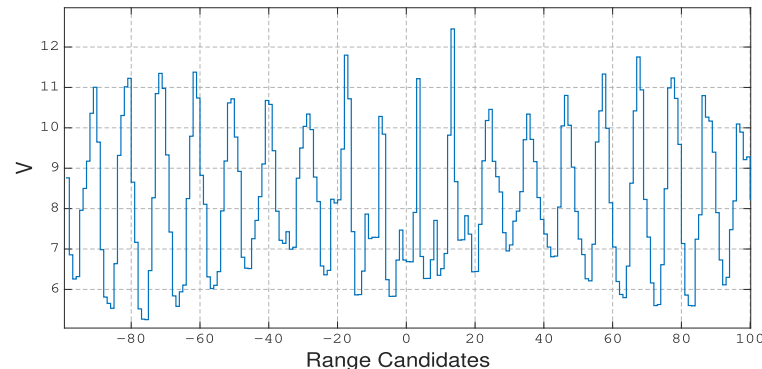

(a)

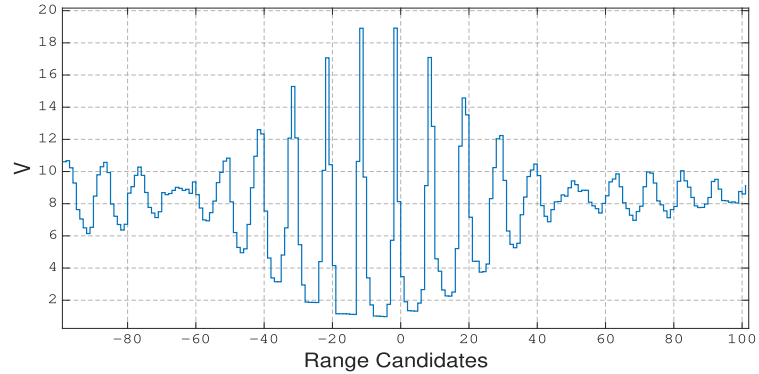

(b)

Fig. 6. Refinement variance $V$ for 200 range candidates where the true value is at -6 . (a) Before Doppler compensation. (b) After Doppler compensation.

within one wavelength, a minimum refinement-variance search algorithm is applied. This algorithm calculates the variance in the estimated refinement $\hat{\Delta} d_{m}$ over the valid frequency bins and at different range candidates.

Let $z_{i}[k]$ be the circularly shifted version of $z[k]$, where the index $i$ represents the amount by which we shift $z[k]$. Each circular shift of $z[k]$ represents a range candidate, where the range candidate associated with zero cyclic shift is $\hat{d}_{\text {corr }}$. Using $z_{i}[k]$ instead of $z[k]$ in (37)-(41) gives the estimated range refinement $\hat{\Delta} d_{i}$ for each range candidate. The refinement variance $V_{i}$ associated with the $i$ th range candidate is given by

$$
V_{i}=\frac{\sum_{m=0}^{M-1}\left(\hat{\Delta} d_{i}-\hat{\Delta} d_{m}\right)^{2}}{M} .
$$

When the absolute difference $\left|d-\hat{d}_{\text {corr }}\right|$ is less than $\lambda_{\max } / 2$, the estimated range refinements $\hat{\Delta} d_{m}$ will be consistent over all the valid frequency bins, resulting in low variance $V_{i}$. In contrast, when the difference $\left|d-\hat{d}_{\text {corr }}\right|$ is larger than $\lambda_{\max } / 2$, the estimated range refinements $\hat{\Delta} d_{m}$ will fluctuate over the valid frequency bins, producing a high variance $V_{i}$. The proposed method estimates the refinement variance $V_{i}$ for different range candidates. The range candidate with the minimum refinement variance over the valid frequency bins is chosen as the correct range estimate. Fig. 6 shows the refinement variance $V_{i}$ for a window of 200 range candidates before and after compensating for the frequency offsets under low SNR $(-10 \mathrm{~dB})$. The initial range estimate $\hat{d}_{\text {corr }}$ has an error of six samples; hence, the location of the minimum variance is at -6 . Therefore, the minimum variance search algorithm enables us to correct the initial range to within one wavelength of the signal.

Whether we use the full-fledged ML receiver or its highSNR-equivalent, the ranging algorithm demonstrated in this

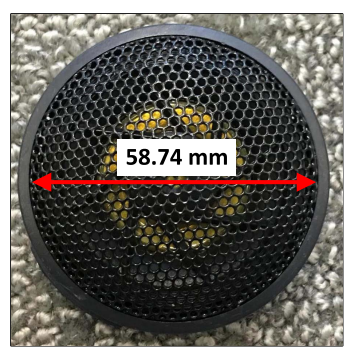

(a)

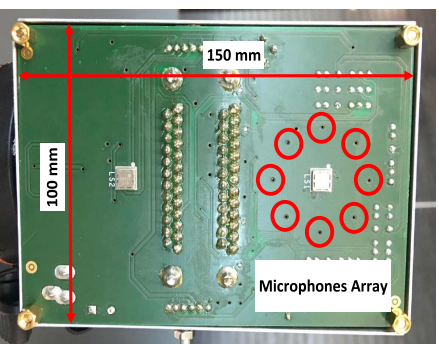

(b)
Fig. 7. Tx and Rx. (a) Pioneer TS-T110 tweeter. (b) Microphones array on a PCB.

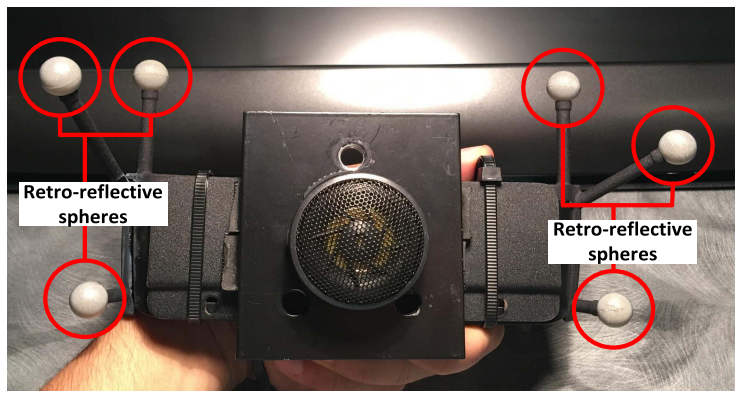

Fig. 8. Transmitter attached to the target object.

section has a much lower complexity. While the performance of the proposed algorithm is slightly degraded compared to the DZC ML estimator, it still outperforms the ZC ML estimator.

\section{EXPERIMENTAL SETUP}

This section describes the experimental setup and the hardware used to test and evaluate the proposed system. We used IQ modulation to transmit the complex signals. In IQ modulation, the real part of the signal is modulated using a cosine wave and the imaginary part is modulated using a sine wave. The transmitter (Tx) used in this setup is a Pioneer TS-T110 tweeter, shown in Fig. 7(a), which has a bandwidth (BW) of $7 \mathrm{kHz}$ and a central frequency of $20 \mathrm{kHz}$. The receiver $(\mathrm{Rx})$ is a microphone on a printed circuit board (PCB) as shown in Fig. 7(b). XLR connectors link the transmitter and the receiver to the computer through a sound card (E44 Express) that provides a sampling rate up to $192 \mathrm{kHz}$. The data are recorded as .wav files during experiments and saved in the computer to be processed offline. The experiments are implemented in a typical indoor environment with dimensions $1000 \mathrm{~cm} \times 800 \mathrm{~cm} \times 400 \mathrm{~cm}$. Due to negligible changes in both temperature and humidity, the speed of sound is assumed to be constant during the experiments $(345.664 \mathrm{~m} / \mathrm{s})$. An infrared tracking system (OptiTrack), which gives the ground truth with $0.1-\mathrm{mm} 3-\mathrm{D}$ accuracy and a maximum update rate of $250 \mathrm{~Hz}$ [31], provides a benchmark for evaluating the performance of the proposed system. The tracking system provides accurate 3-D location and orientation information via a set of infrared cameras (up to 16 cameras) that keep track of small spheres coated with retro-reflective film. Fig. 8 shows the target tracked by the IR cameras with the transmitter attached to it. Fig. 9 shows the experimental setup. 


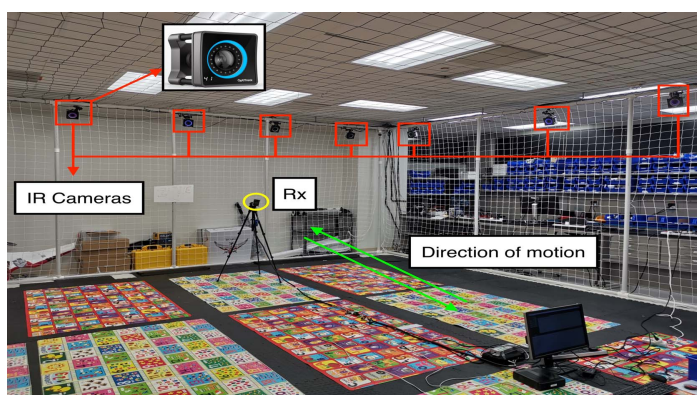

(a)

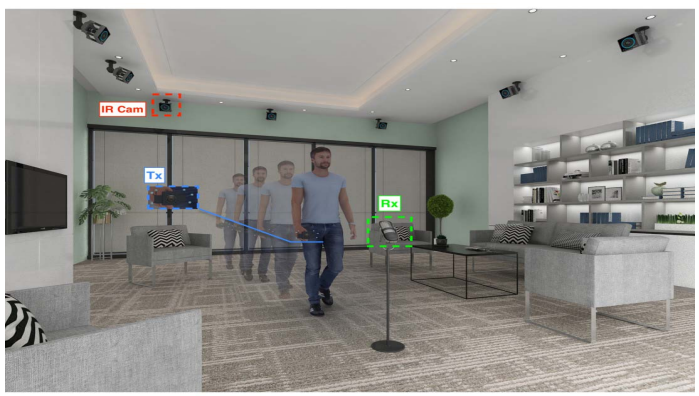

(b)

Fig. 9. Experimental setup. (a) Actual setup. (b) Illustration.

TABLE I

RUNNING TIME (ms) FOR ONE ITERATION OF THE RANGE ESTIMATION ALGORITHM

\begin{tabular}{|c|c|c|c|}
\hline $\begin{array}{c}\text { Tx duration } \\
\text { (ms) }\end{array}$ & $\begin{array}{c}\text { MUSIC-based LSE [22] } \\
\text { (DZC) }\end{array}$ & $\begin{array}{c}\text { Super-res radar [23] } \\
\text { (DZC) }\end{array}$ & $\begin{array}{c}\text { Diff Corr } \\
\text { (DZC) }\end{array}$ \\
\hline 6 & 1573 & 4781 & 3.99 \\
\hline
\end{tabular}

\section{RESUlts}

\section{A. Simulation Results}

In this section, we compare different TOF estimation algorithms of a moving target using the same DZC sequence. All simulations were performed using MATLAB. Unless stated otherwise, the simulation results in this section were obtained from 10000 samples. We compare the RMSE of the TOF estimates under a constant Doppler shift. We estimate the TOF using a MUSIC-based LSE algorithm [22], a superresolution radar, via solving $\ell_{1}$ minimization program [23], the proposed maximum likelihood estimator (MLE), and the proposed reduced-complexity algorithm. Fig. 10 shows the RMSEs of the TOF estimates under different SNR scenarios (with $N=21, \tilde{\tau}=10$ symbols duration, $M=1$, and $\widetilde{v}=1$ ). In both low and high SNR scenarios, the MLE outperforms all the other algorithms. In a high SNR scenario, the proposed reduced-complexity ranging algorithm outperforms both the MUSIC-based LSE algorithm [22] and the super-resolution-radar estimation algorithm [23]. In addition, as shown in Table I, the proposed reduced-complexity algorithm requires a much lower computational cost than the MUSIC-based LSE and the super-resolution-radar algorithms. Because of the high computational complexity of the latter two algorithms, we chose $N=21$ in this comparison.

Next, using DZC sequences, we compare the performance of the differential-sliding-correlation-based ranging algorithm, against three other algorithms: the STFT on FMCW [17],

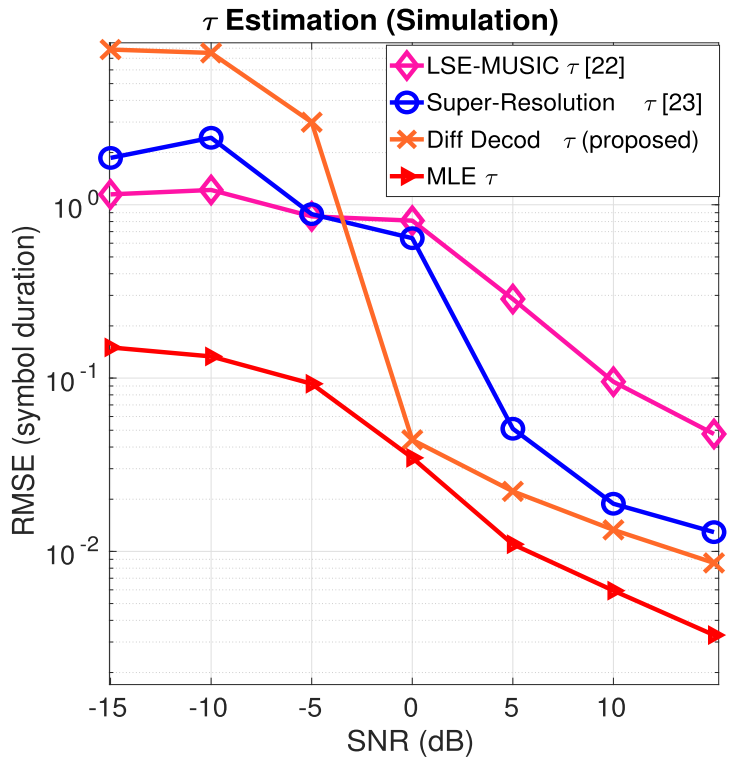

Fig. 10. TOF estimation using MLE, LSE, and reduced-complexity algorithm with $N=21, \widetilde{\tau}=10$ symbols duration, $M=1$, and $\widetilde{v}=1$.

TABLE II

RunNing Time (ms) OF the Four RANGing ALgorithms

\begin{tabular}{|c|c|c|c|}
\hline $\begin{array}{c}\text { Tx duration } \\
(\mathrm{ms})\end{array}$ & $\begin{array}{c}\text { Maximum Likelihood } \\
\text { (Diff or regular ZC) }\end{array}$ & $\begin{array}{c}\text { STFT } \\
\text { (FMCW) }\end{array}$ & $\begin{array}{c}\text { Diff Corr } \\
\text { (Diff ZC) }\end{array}$ \\
\hline 120 & 1022 & 595 & 54 \\
\hline
\end{tabular}

the $\mathrm{ML}$ estimator using regular $\mathrm{ZC}$ sequences, and the $\mathrm{ML}$ estimator using DZC sequences, derived in Section IV. For each of the four algorithms, a signal with a duration of $120 \mathrm{~ms}$ is generated. Using MATLAB, a moving target with a velocity of $1 \mathrm{~m} / \mathrm{s}$ is simulated. The received signal is composed of the delayed and Doppler-shifted transmitted signal with additive white Gaussian noise (AWGN) under a random phase shift. The RMSEs of the estimated TOFs from each of the four algorithms are shown in Fig. 11. In this simulation, we use a sampling frequency of $192 \mathrm{kHz}$, so each sample represents around $1.78 \mathrm{~mm}$. Fig. 11 shows that the RMSE from the ML estimation with the DZC sequences is lower than the RMSE with the regular $\mathrm{ZC}$ sequences, meaning that $\mathrm{ML}$ estimation with DZC sequences can achieve higher accuracy than with regular $\mathrm{ZC}$ sequences.

Moreover, under high SNR scenarios, the differentialsliding-correlation of the DZC sequences outperforms the ML estimation using regular ZC sequences. Under low SNR conditions, ML estimation performs the best out of the four algorithms. Furthermore, using DZC sequences, the differential-sliding-correlation ranging algorithm outperforms the FMCW ranging algorithm [17]. Table II shows the running time for each of the four algorithms to estimate the range. From this table, we can see that the differentialsliding-correlation has the lowest computational complexity from among the four algorithms.

We run more simulations to evaluate the performance of the proposed algorithm for different sequence lengths. Table III shows the RMSE of the proposed algorithm for various sequence lengths at a fixed velocity of $0.1 \mathrm{~m} / \mathrm{s}$ and different 


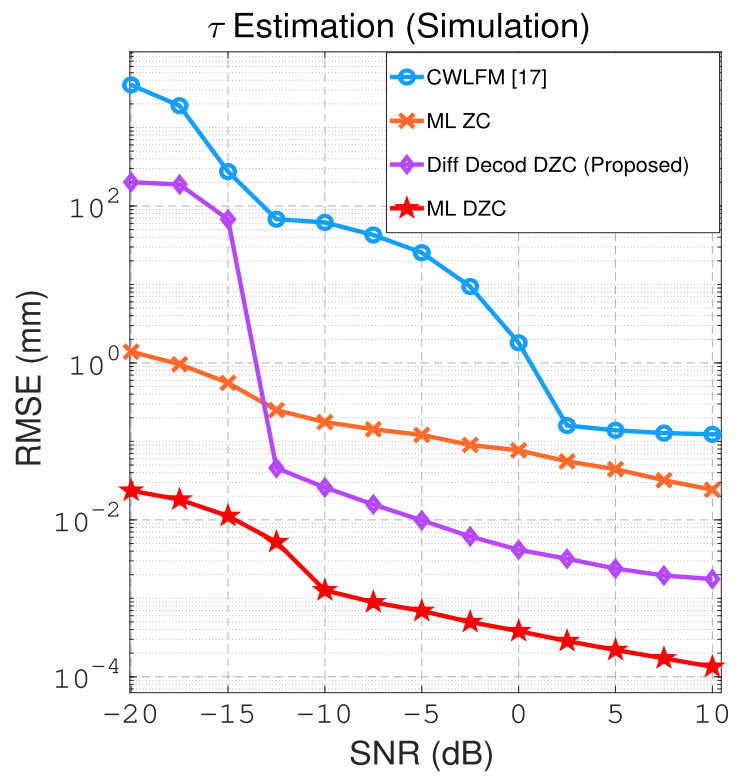

Fig. 11. Simulation results: RMSE (in (mm) for the normalized TOF $\tau$ ) versus SNR for a signal duration of $120 \mathrm{~ms}$, TOF $\tau=60 \mathrm{~ms}$.

TABLE III

RMSE For DifFERENT LENGTHS With A FiXed Velocity $0.1 \mathrm{~m} / \mathrm{s}$

\begin{tabular}{|c|c|c|c|c|}
\hline \multirow[b]{2}{*}{$\begin{array}{c}\text { Length } \\
\text { (symbols) }\end{array}$} & $\mathrm{SNR}=20 \mathrm{~dB}$ & $10 \mathrm{~dB}$ & $0 \mathrm{~dB}$ & $-10 \mathrm{~dB}$ \\
\hline & $\begin{array}{c}\text { RMSE } \\
(\mathrm{mm})\end{array}$ & $\begin{array}{c}\text { RMSE } \\
(\mathrm{mm})\end{array}$ & $\begin{array}{c}\text { RMSE } \\
(\mathrm{mm})\end{array}$ & $\begin{array}{c}\text { RMSE } \\
(\mathrm{mm})\end{array}$ \\
\hline 15 & 1.654 & 1.654 & 3.104 & 6.926 \\
\hline 31 & 0.743 & 0.923 & 1.958 & 6.576 \\
\hline 63 & 0.489 & 0.489 & 1.508 & 4.175 \\
\hline 255 & 0.480 & 0.558 & 0.651 & 2.050 \\
\hline 511 & 0.282 & 0.282 & 0.282 & 0.916 \\
\hline
\end{tabular}

TABLE IV

RMSE OF DIFFERENTIAL CORRELATION RANGING FOR DIFFERENT VELOCITIES AND DIFFERENT BWs $(N=511$ sYMBOLS, $\mathrm{SNR}=5 \mathrm{~dB})$

\begin{tabular}{|c|c|c|c|}
\hline & $\mathrm{BW}=19.2 \mathrm{kHz}$ & $6.4 \mathrm{kHz}$ & $4.8 \mathrm{kHz}$ \\
\hline $\begin{array}{l}\text { Velocity } \\
(\mathrm{m} / \mathrm{s})\end{array}$ & $\begin{array}{l}\text { RMSE } \\
(\mathrm{mm})\end{array}$ & $\begin{array}{c}\text { RMSE } \\
(\mathrm{mm})\end{array}$ & $\begin{array}{c}\text { RMSE } \\
(\mathrm{mm})\end{array}$ \\
\hline 0.51 & 3.339 & 1.992 & 0.759 \\
\hline 1.01 & 6.960 & 4.875 & 3.623 \\
\hline 1.51 & 10.629 & 9.437 & 7.619 \\
\hline 2.01 & 14.943 & 14.976 & 12.542 \\
\hline
\end{tabular}

SNR values. The results are obtained from 10000 observations. Short sequences, e.g., with $N=15$ and $N=31$, have lower energy and therefore are more susceptible to noise than longer sequences, e.g., with $N=255$ and $N=511$.

Next, we evaluate the performance of our algorithm under various velocities and for different BWs (from 10000 samples with a fixed SNR $=5 \mathrm{~dB}$ ). Table IV shows the RMSE for the differential-correlation ranging algorithm using a 511-symbol DZC sequence and different BWs and target velocities. As expected, the RMSE increases with the velocity as well as with a higher BW. We attribute the increase in the RMSE with the higher BW to the narrowband approximation of the received signal when using differential correlation, as in (3) (i.e., a lower BW means our approximation is more accurate).

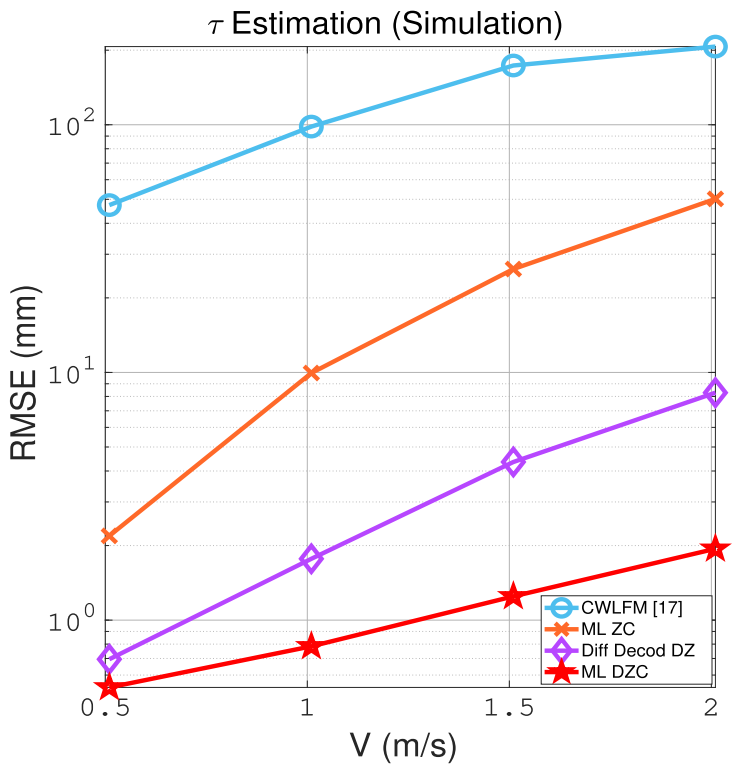

Fig. 12. RMSE for different velocities with $\mathrm{SNR}=20 \mathrm{~dB}$ and $\mathrm{BW}=6.4 \mathrm{kHz}$.

Then, using several algorithms, we evaluate the performance of different codes under various velocities at a fixed BW of $6.4 \mathrm{kHz}$ and a fixed SNR of $20 \mathrm{~dB}$. As can be seen in Fig. 12, the proposed algorithm outperforms the benchmark codes and algorithms at a range of velocities.

\section{B. Experimental Results}

This section presents the experimental results to evaluate the proposed system. In our experimental setup, an ultrasound receiver on a PCB is fixed in a specific location, while the ultrasound transmitter is moving. A set of infrared cameras provides the location of the moving transmitter with submillimeter accuracy. The motion ground truth is found by converting the positions of both the transmitter and the receiver into ranges. Each of the three signals was periodically transmitted over a total duration of around $30 \mathrm{~s}$, with the transmitter stationary in the first few seconds, then moving forward and backward before stopping at a specified distance for the last few seconds.

Our first experiment demonstrates the limitations of the regular ZC cross correlation-based ranging, in which a set of 511-symbol ZC sequences is used to track the moving transmitter. When the transmitter is stationary, the estimated range is accurate. However, when the transmitter is moving, the ranging accuracy degrades significantly due to the Doppler effect. Fig. 13(a) shows clearly the accuracy degradation from Doppler in the estimated range using the regular $\mathrm{ZC}$ sequence. Our second experiment validates the ability of the proposed DZC to combat the Doppler effect. In this experiment, a set of 511-symbol DZC sequences is used to track the moving transmitter with a velocity similar to the first experiment. Because we are dealing with human motion and not robots, it is impossible to maintain the exact same speed in all experiments, so the speeds in the two experiments are similar but not identical. Fig. 13(b) illustrates the ability of the 


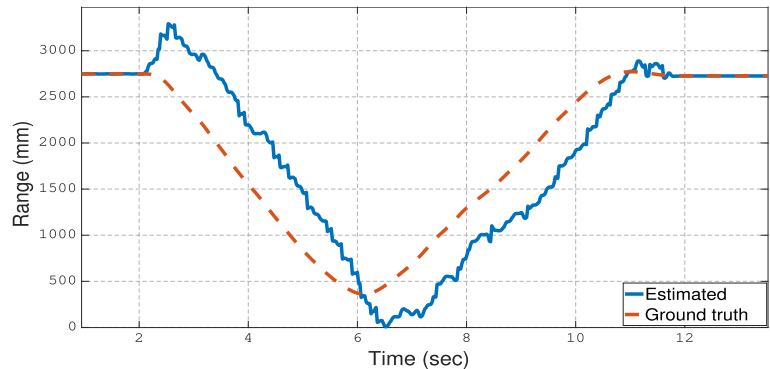

(a)

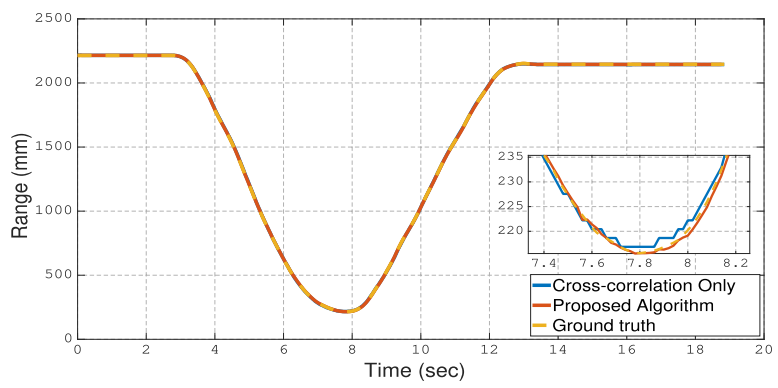

(b)

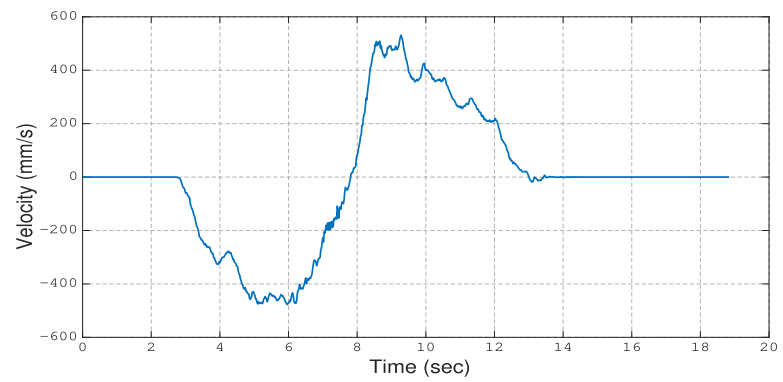

(c)

Fig. 13. Cross correlation-based range estimation using (a) 511-ZC sequence, (b) 511-DZC sequence, and (c) velocity of the moving target.

TABLE V

Estimated RANGe RMSE fOR a MOVIng TRANSMitTER

\begin{tabular}{|c|c|c|}
\hline $\begin{array}{c}\text { Sequence Length } \\
\text { (symbols) }\end{array}$ & ZC Correlation-based & Reduced-complexity \\
\cline { 2 - 3 } & $\begin{array}{c}\text { RMSE } \\
(\mathrm{mm})\end{array}$ & $\begin{array}{c}\text { RMSE } \\
(\mathrm{mm})\end{array}$ \\
\hline 511 & 319.9687 & 0.7586 \\
\hline
\end{tabular}

DZC sequence to track movements without being affected by Doppler. Fig. 13(c) shows the velocity of the moving target during the experiments. The results in Fig. 13 were obtained over 41600 samples. Table V shows the RMSE of the estimated range in those two experiments.

The recorded data are processed under different SNR values by adding white Gaussian noise to the data in MATLAB to validate the minimum refinement-variance search algorithm. Fig. 14 shows the percentage of range estimates that are correct up to half the wavelength of the maximum frequency (around $7.5 \mathrm{~mm}$ ). As the SNR goes lower, the accuracy of the estimated ranges without the minimum refinement-variance search degrades, while the accuracy of the estimates obtained using the minimum refinement-variance search stays high. Fig. 15(a)-(d) show the cumulative error under different SNR values with and without the minimum refinement-variance search algorithm. Using the minimum refinement-variance

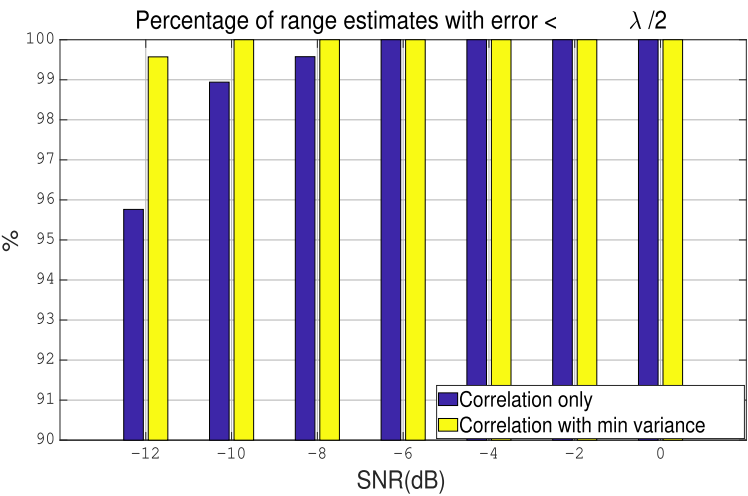

Fig. 14. Percentage of estimates correct up to $\lambda_{\min } / 2$.

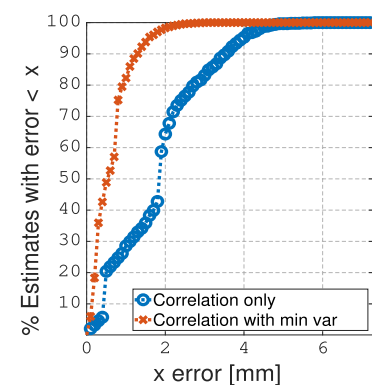

(a)

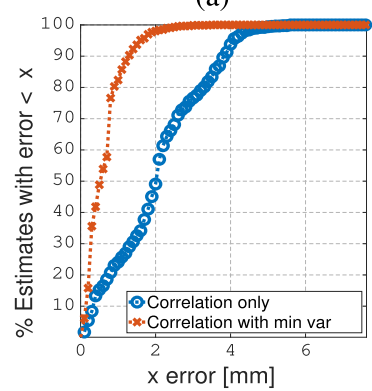

(c)

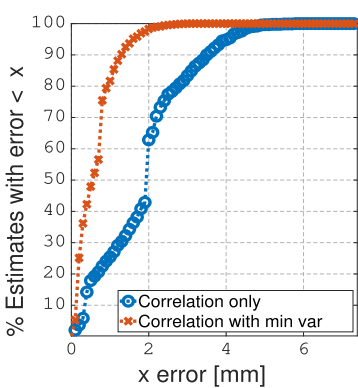

(b)

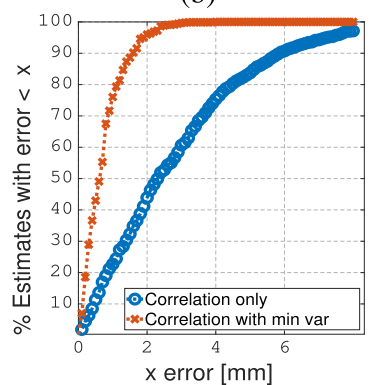

(d)
Fig. 15. Cumulative error in range estimates at (a) $20 \mathrm{~dB}$, (b) $10 \mathrm{~dB}$, (c) $0 \mathrm{~dB}$, and (d) $-10 \mathrm{~dB}$ SNR using 511-symbol sequence.

\author{
TABLE VI
}

RMSE OF RANGE ESTIMATES FOR A MOVING TRANSMITTER USING A 511-SYMBOL SEQUENCE

\begin{tabular}{|c|c|c|}
\hline SNR & diff-correlation only & Reduced-complexity Algorithm \\
\hline$(\mathrm{dB})$ & $\begin{array}{c}\text { RMSE } \\
(\mathrm{mm})\end{array}$ & $\begin{array}{c}\text { RMSE } \\
(\mathrm{mm})\end{array}$ \\
\hline 20 & 2.1407 & 0.7467 \\
\hline 10 & 2.1886 & 0.7585 \\
\hline 0 & 2.3770 & 0.7794 \\
\hline-10 & 3.6127 & 0.8434 \\
\hline
\end{tabular}

search algorithm always improves the accuracy of the estimated range.

Table VI shows the RMSE of the estimated ranges under different SNR values employing only differential correlation, and the reduced-complexity ranging algorithm. The reducedcomplexity algorithm uses differential correlation with the minimum refinement-variance search and phase shift compensation to reach subsample accuracy. The phase refinement improves the initial range estimates to very high accuracy levels even under the low SNR scenario. 


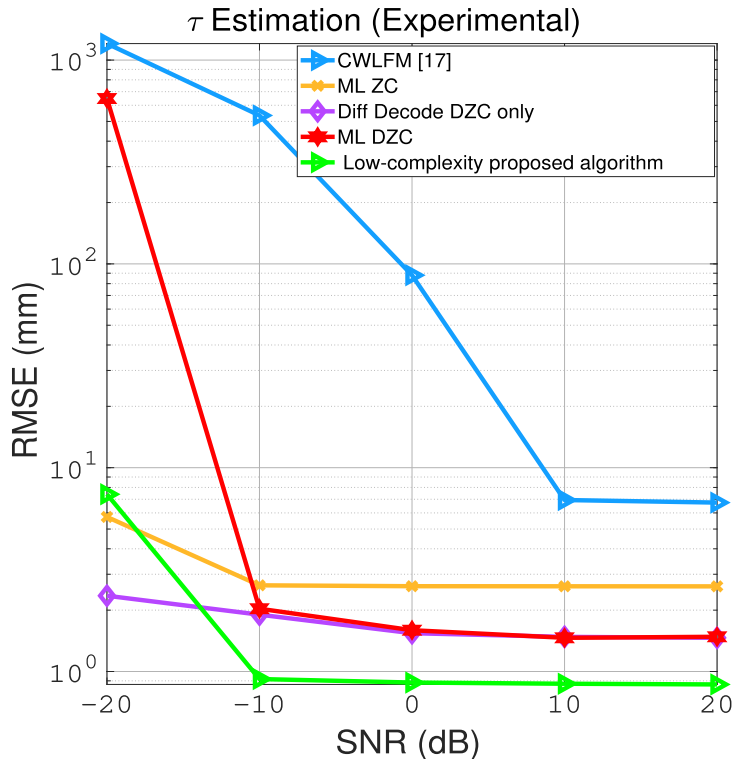

Fig. 16. Experimental results: RMSE (in (mm) for the normalized TOF $\tau$ ) versus SNR for a signal duration of $120 \mathrm{~ms}$.

Using the three types of signals and three algorithms, Fig. 16 shows the RMSE of the TOF estimation under different SNR scenarios with a maximum velocity of $0.5 \mathrm{~m} / \mathrm{s}$. The accuracy of the TOF estimation is dictated by the hardware BW and sampling rate, which in turn control the ranging resolution of the system. The ranging resolution of our experimental setup is around $1.78 \mathrm{~mm} / \mathrm{sample}$. Any movement that is equivalent to a fraction of a sample is rounded to the nearest integer multiple of the sampling resolution. The phase-refinement algorithm compensates for this fractional TOF, improving the ranging accuracy for this method over the others. Moreover, the minimum refinement-variance search shows the robustness of the reduced-complexity algorithm under low SNR conditions compared to the other algorithms. Finally, we would like to highlight that the experimental accuracy of the DZC ML-based ranging can be improved if we perform our grid search with a subsample resolution. However, this has a very high computational cost and thus is difficult to implement. We would like to highlight that the slight degradation in the performance of the proposed algorithm versus the other algorithms in the low SNR scenario is due to the nature of the proposed differential decoding. By applying the differential decoding we double the noise, and therefore, under extremely low SNR values, the accuracy degrades.

Next, using different sequence lengths, we evaluate the performance of the proposed algorithm under different SNR values. Table VII shows the RMSE evaluated experimentally under different lengths, for the proposed algorithm. The results in Table VII are obtained from around 41600 samples under different velocities, with a maximum velocity of $0.53 \mathrm{~m} / \mathrm{s}$. The velocity of the moving target is shown in Fig. 13(c). The experimental results show that, with increasing sequence lengths, the algorithm becomes more robust to noise. We note that, except at $0 \mathrm{~dB} \mathrm{SNR}$, the performance of the proposed algorithm using a sequence of 31 symbols is worse than its performance with a sequence of 15 symbols. While this is not

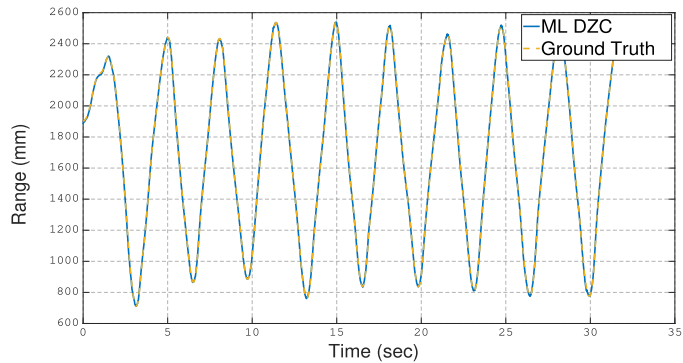

(a)

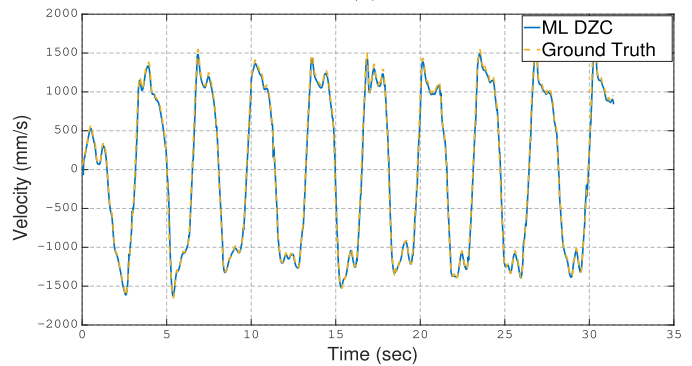

(b)

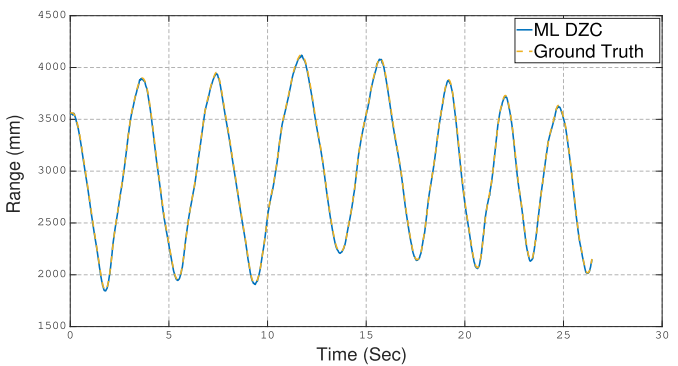

(c)

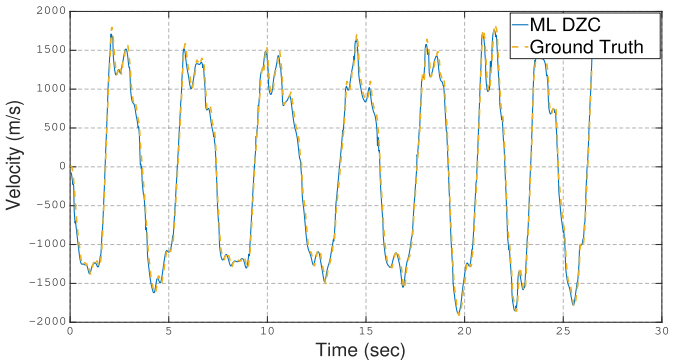

(d)

Fig. 17. ML-based DZC estimated range and velocity with max velocity $1.115 \mathrm{~m} / \mathrm{s}$ in (a) and (b) and $1.911 \mathrm{~m} / \mathrm{s}$ in (c) and (d).

TABLE VII

EXPERIMENTAL: RMSE FOR THE REDUCED-COMPLEXITY ALGORITHM USING DiFFERENT LENGTHS UNDER DIFFERENT SNR VALUES

\begin{tabular}{|c|c|c|c|c|}
\hline \multirow[b]{2}{*}{$\begin{array}{c}\text { Length } \\
\text { (symbols) }\end{array}$} & $\mathrm{SNR}=20 \mathrm{~dB}$ & $10 \mathrm{~dB}$ & $0 \mathrm{~dB}$ & $-10 \mathrm{~dB}$ \\
\hline & $\begin{array}{l}\text { RMSE } \\
(\mathrm{mm})\end{array}$ & $\begin{array}{c}\text { RMSE } \\
(\mathrm{mm})\end{array}$ & $\begin{array}{c}\text { RMSE } \\
(\mathrm{mm})\end{array}$ & $\begin{array}{c}\text { RMSE } \\
(\mathrm{mm})\end{array}$ \\
\hline 15 & 0.943 & 0.944 & 59.32 & 494.9 \\
\hline 31 & 1.394 & 1.393 & 40.13 & 871.4 \\
\hline 63 & 0.8728 & 0.8786 & 9.212 & 21.63 \\
\hline 255 & 0.8593 & 0.8557 & 0.8521 & 10.03 \\
\hline 511 & 0.7466 & 0.7583 & 0.7791 & 0.8432 \\
\hline
\end{tabular}

expected, this might be attributed to some outliers during the experiment.

Finally, we evaluate the proposed algorithm and code against the benchmark algorithms for different velocities at a fixed SNR value $(10 \mathrm{~dB})$. In each experiment, the velocity 
TABLE VIII

EXPERIMENTAL: RMSE FOR THE PROPOSED AND BENCHMARK ALGORITHMS UNDER DIFFERENT VELOCITIES

\begin{tabular}{|c|c|c|c|c|}
\hline \multirow[b]{2}{*}{$\begin{array}{c}\text { Max Velocity } \\
(\mathrm{m} / \mathrm{s})\end{array}$} & ML ZC & CWLFM [17] & Diff DZC & ML DZC \\
\hline & $\begin{array}{c}\text { RMSE } \\
(\mathrm{mm})\end{array}$ & $\begin{array}{c}\text { RMSE } \\
(\mathrm{mm})\end{array}$ & $\begin{array}{c}\text { RMSE } \\
(\mathrm{mm})\end{array}$ & $\begin{array}{c}\text { RMSE } \\
(\mathrm{mm})\end{array}$ \\
\hline 0.915 & 73.95 & 61.51 & 9.520 & 4.547 \\
\hline 1.115 & 566.8 & 111.1 & 16.39 & 7.627 \\
\hline 1.911 & 335.8 & 665.6 & 24.02 & 7.696 \\
\hline
\end{tabular}

of the moving target varies with time. Table VIII shows the RMSE for the proposed and benchmark algorithms under different velocities. The proposed DZC sequences and ranging algorithms outperform the benchmark ranging algorithms. The performance of the ZC-based ML-ranging algorithm degrades because of the ambiguity of jointly estimating the TOF and the Doppler shift. Fig. 17 shows the estimated range and velocity of a moving target using the proposed ML-based DZC ranging algorithm in two experiments. In Fig. 17(a) and (b), the maximum velocity of the moving target is $1.115 \mathrm{~m} / \mathrm{s}$, and in Fig. 17(c) and (d), the maximum velocity is $1.911 \mathrm{~m} / \mathrm{s}$, demonstrating that the proposed algorithm and signal design provide high ranging accuracy even at high velocities.

\section{CONCLUSION}

In this article, we present a novel signal design for estimating the range of a moving transmitter based on the differential coding of ZC sequences. We evaluate the proposed DZC design and ranging algorithms, both in simulation and using an experimental setup in a typical indoor environment. In both the simulations and the real experiments, the proposed DZC design outperforms the regular ZC sequences in estimating both the TOF and the Doppler shift of a moving target. Moreover, our simulation results show that, using a DZC sequence, the proposed differential-sliding-correlation outperforms the benchmark algorithms-namely STFT-based range estimation with an FMCW and ML range estimation with a regular ZC sequence. Compared to the benchmark algorithms, the differential-sliding-correlation has the lowest computational complexity when estimating the range of a moving target.

The experimental results show that, while the typical long $\mathrm{ZC}$ sequence fails to estimate the range of a moving transmitter using cross correlation, the proposed DZC sequence is able to combat the Doppler effect by utilizing the differentialsliding-correlation. Even in low SNR scenarios, the minimum refinement-variance search algorithm is able to provide robustness to the system by correcting range estimates up to half the wavelength of the signal carriers. At low SNR $(-12 \mathrm{~dB})$, the minimum refinement-variance search algorithm reduces the error of $98 \%$ of the range estimates to less than half the wavelength of the signal carriers, compared to $92 \%$ when not using the algorithm.

The proposed low-complexity ranging algorithm, using a DZC sequence of 511 symbols, has an RMSE of $0.75 \mathrm{~mm}$ at an SNR of $20 \mathrm{~dB}$, and an RMSE of $0.84 \mathrm{~mm}$ at an SNR of $-10 \mathrm{~dB}$, which shows the capacity of the proposed system to achieve subsample resolution, even in low SNR scenarios. Moreover, as expected, the experimental results show that the low-complexity ranging and ML-based ranging algorithms with DZC sequences outperform the ML-based ZC ranging and the FMCW STFT-based ranging algorithms.

The proposed ranging system can be employed in various applications that require estimating the position of a moving target. One such system that requires high positioning accuracy is a robotic surgical system. Another widely used ranging application is indoor localization, which requires estimating the distances between a receiver, known as the mobile device, and multiple transmitters, known as base-stations. These basestations need to be synchronized, ideally by transmitting an RF signal with the ultrasound signal. After determining the distance between each base-station and the target, we can use a trilateration algorithm to find the position of the target. In indoor navigation applications, we can extend our current work to localize multiple users by giving each user a unique DZC code (i.e., a DZC code with a unique DZC exponent $M$ ). Each DZC code has an excellent auto-correlation property and poor cross correlation with the other DZC codes, thus providing orthogonality between the various users. Moreover, the proposed ranging algorithm can be used in systems with low sampling rates, in which the ranging resolution is dictated by the sampling frequency, so the phase refinement can significantly improve the resolution of such systems.

\section{APPENDIX A}

\section{Analytical Derivation of DZC Sequence Design}

Applying the differential-decoding to a DZC sequence $a[k]$ gives a ZC sequence $s[k]$ in which the phase is a quadratic function in time. Therefore, the phase of the DZC sequence is a cubic function in time

$$
a^{*}[k] a[k+1]=s[k]
$$

where $a[k]=e^{j\left(\alpha_{3} k^{3}+\alpha_{2} k^{2}+\alpha_{1} k+\alpha_{0}\right)}$. For an even-length ZC sequence, (A.44) gives

$$
\begin{aligned}
& -\left(\alpha_{3} k^{3}+\alpha_{2} k^{2}+\alpha_{1} k+\alpha_{0}\right)+\left(\alpha_{3}(k+1)^{3}+\alpha_{2}(k+1)^{2}\right. \\
& \left.+\alpha_{1}(k+1)+\alpha_{0}\right)=\frac{M \pi k^{2}}{N} \\
& \alpha_{3}\left(3 k^{2}+3 k+1\right)+\alpha_{2}(2 k+1)+\alpha_{1}=\frac{M \pi k^{2}}{N} .
\end{aligned}
$$

Solving (A.45) results in $\alpha_{3}=(M \pi / 3 N), \alpha_{2}=(-M \pi / 2 N)$, $\alpha_{1}=(M \pi / 6 N)$, and $\alpha_{0}$ is an arbitrary constant which is set to zero for simplicity. The phase of the DZC $\phi[k]$ becomes

$$
\begin{aligned}
\phi[k] & =\alpha_{3} k^{3}+\alpha_{2} k^{2}+\alpha_{1} k+\alpha_{0} \\
& =\frac{M \pi}{3 N} k^{3}-\frac{M \pi}{2 N} k^{2}+\frac{M \pi}{6 N} k \\
& =\frac{M \pi}{3 N} k\left(k-\frac{1}{2}\right)(k-1) .
\end{aligned}
$$

For an odd-length ZC sequence, (A.44) gives

$\alpha_{3}\left(3 k^{2}+3 k+1\right)+\alpha_{2}(2 k+1)+\alpha_{1}=\frac{M \pi\left(k^{2}+k\right)}{N}$. 
Solving (A.46) results in $\alpha_{3}=(M \pi / 3 N), \alpha_{2}=0, \alpha_{1}=$ $-(M \pi / 3 N)$, and $\alpha_{0}$ is an arbitrary constant which is set to zero for simplicity. The phase of the DZC $\phi[k]$ becomes

$$
\begin{aligned}
\phi[k] & =\alpha_{3} k^{3}+\alpha_{2} k^{2}+\alpha_{1} k+\alpha_{0} \\
& =\frac{M \pi}{3 N} k^{3}-\frac{M \pi}{3 N} k \\
& =\frac{M \pi}{3 N} k(k-1)(k+1) .
\end{aligned}
$$

\section{APPENDIX B \\ PERIODICITY OF DZC CODES}

For repetitive and periodic transmission, each sequence is repeated $P$ times, where $P$ is an integer and each single sequence is referred to as a block. It is important to determine the period of the DZC sequence to perform the periodic transmission.

For an even-length DZC sequence, with all the phases to be taken modulo $2 \pi$, adding a shift of $N$ symbols in time gives

$$
\begin{aligned}
\phi_{k+N}= & \frac{\pi M}{3 N}(k+N)(k+N-1)\left(k+N-\frac{1}{2}\right) \\
= & \frac{\pi M}{3 N} k(k-1)\left(k-\frac{1}{2}\right)+\pi M \underbrace{k(k+(N-1))}_{\text {even for even } N} \\
& +\frac{\pi M}{3}(N-1)\left(N-\frac{1}{2}\right) \\
= & \phi_{k}+\frac{\pi M}{3}\left(N-\frac{1}{2}\right)(N-1) .
\end{aligned}
$$

Therefore, shifting $a[k]$ by $N$ results in the same sequence $a[k]$ but with a phase shift of $(\pi M / 6)(2 N-1)(N-1)$ up to an additive integer multiple of $2 \pi$. If $N$ is even and either $(2 N-1)$ or $(N-1)$ is a multiple of 3 , then the period of the sequence is $4 N$. Otherwise, the period is $12 N$.

Similarly considering an odd-length DZC sequence, with all the phases to be taken modulo $2 \pi$ gives

$$
\begin{aligned}
\phi_{k+N}= & \frac{\pi M}{3 N}(k+N)(k+N-1)(k+N+1) \\
= & \frac{\pi M}{3 N} k(k-1)(k+1)+\pi M \underbrace{k(k+N)}_{\text {even for odd } N} \\
& +\frac{\pi M}{3}\left(N^{2}-1\right) \\
= & \phi_{k}+\frac{\pi M}{3}(N+1)(N-1) .
\end{aligned}
$$

Therefore, shifting $a[k]$ by $N$ results in the same sequence as $a[k]$ but with a phase shift of $(\pi M / 3)(N+1)(N-1)$ up to an additive integer multiple of $2 \pi$. If $N$ is odd and not divisible by 3 , then either $(N-1)$ or $(N+1)$ is even and divisible by 3 , hence the sequence period is $N$. Otherwise, if $N$ is odd and divisible by 3 then the sequence period is $3 N$, which can be written as follows:

$$
\begin{aligned}
\phi_{k+3 N} & =\phi_{k+2 N}+\frac{\pi M}{3}\left(N^{2}-1\right) \\
& =\phi_{k+N}+\frac{\pi M}{3}\left(N^{2}-1\right)+\frac{\pi M}{3}\left(N^{2}-1\right) \\
& =\phi_{k}+\pi M\left(N^{2}-1\right) .
\end{aligned}
$$

Since $N$ is odd, the term $\left(N^{2}-1\right)$ is even, taking modulo $2 \pi$ gives

$$
\phi_{k+3 N}=\phi_{k} .
$$

To sum up, the DZC sequence is given by

$$
a[k]=e^{i \phi[k]}
$$

where

$$
\phi[k]= \begin{cases}\frac{\pi M}{3 N} k\left(k-\frac{1}{2}\right)(k-1), & k=1,2, \ldots, 2 N\left(2+a_{1} a_{2}\right), \\ \frac{\pi M}{3 N} k(k+1)(k-1), & \text { for even } N \\ & \text { for odd } N\end{cases}
$$

where $a_{1}=((2 N-1)$ modulo 3$), a_{2}=((N-1)$ modulo 3$)$, and $a_{3}=(N$ modulo 3$)$.

\section{REFERENCES}

[1] J. Hightower and G. Borriello, "A survey and taxonomy of location systems for ubiquitous computing," IEEE Comput., vol. 34, no. 8, pp. 57-66, 2001.

[2] K. Whitehouse, C. Karlof, and D. Culler, "A practical evaluation of radio signal strength for ranging-based localization," ACM SIGMOBILE Mobile Comput. Commun. Rev., vol. 11, no. 1, pp. 41-52, Jan. 2007.

[3] C. C. Yüzbaşioğlu and B. Barshan, "Improved range estimation using simple infrared sensors without prior knowledge of surface characteristics," Meas. Sci. Technol., vol. 16, no. 7, p. 1395, 2005.

[4] M.-C. Amann, T. Bosch, M. Lescure, R. Myllyla, and M. Rioux, "Laser ranging: A critical review of usual techniques for distance measurement," Opt. Eng., vol. 40, no. 1, pp. 10-19, 2001.

[5] R. H. Rasshofer and K. Gresser, "Automotive radar and lidar systems for next generation driver assistance functions," Adv. Radio Sci., vol. 3, pp. 205-209, May 2005.

[6] M. Cypriani, F. Lassabe, P. Canalda, and F. Spies, "Open wireless positioning system: A Wi-Fi-based indoor positioning system," in Proc. IEEE 70th Veh. Technol. Conf. Fall, Sep. 2009, pp. 1-5.

[7] J.-Y. Lee and R. A. Scholtz, "Ranging in a dense multipath environment using an UWB radio link," IEEE J. Sel. Areas Commun., vol. 20, no. 9, pp. 1677-1683, Dec. 2002.

[8] R. M. Narayanan and M. Dawood, "Doppler estimation using a coherent ultrawide-band random noise radar," IEEE Trans. Antennas Propag., vol. 48, no. 6, pp. 868-878, Jun. 2000.

[9] M. Kushwaha, K. Molnar, J. Sallai, P. Volgyesi, M. Maroti, and A. Ledeczi, "Sensor node localization using mobile acoustic beacons," in Proc. IEEE Int. Conf. Mobile Adhoc Sensor Syst. Conf., Nov. 2005, pp. $9-17$.

[10] (2020). Pozyx System Overview. [Online]. Available: https://www. pozyx.io/

[11] (2020). Marvel Mind System Overview. [Online]. Available: https://marvelmind.com/

[12] M. I. Skolnik, Introduction to Radar Systems. New York, NY, USA: McGraw-Hill Book, 1980. p. 590.

[13] C. Medina, J. C. Segura, and S. Holm, "Feasibility of ultrasound positioning based on signal strength," in Proc. Int. Conf. Indoor Positioning Indoor Navigat. (IPIN), Nov. 2012, pp. 1-9.

[14] F. Figueroa and E. Barbieri, "An ultrasonic ranging system for structural vibration measurements," IEEE Trans. Instrum. Meas., vol. 40, no. 4, pp. 764-769, Aug. 1991.

[15] C. F. Huang, M. S. Young, and Y. C. Li, "Multiple-frequency continuous wave ultrasonic system for accurate distance measurement," Rev. Sci. Instrum., vol. 70, no. 2, pp. 1452-1458, Feb. 1999.

[16] M. H. AlSharif, M. Saad, M. Siala, T. Ballal, H. Boujemaa, and T. Y. Al-Naffouri, "Zadoff-Chu coded ultrasonic signal for accurate range estimation," in Proc. 25th Eur. Signal Process. Conf. (EUSIPCO), Aug. 2017, pp. 1250-1254.

[17] W. Mao, J. He, and L. Qiu, "CAT: High-precision acoustic motion tracking," in Proc. 22nd Annu. Int. Conf. Mobile Comput. Netw., Oct. 2016, pp. 69-81. 
[18] S. M. Kay, Fundamentals of Statistical Signal Processing: Practical Algorithm Development, vol. 3. London, U.K.: Pearson Education, 2013.

[19] P. Closas, C. Fernández-Prades, and J. A. Fernández-Rubio, "Maximum likelihood estimation of position in GNSS," IEEE Signal Process. Lett., vol. 14, no. 5, pp. 359-362, May 2007.

[20] S. K. Han et al., "Frequency ambiguity free tiered differential-polyphase codes for GNSS signal design," Electron. Lett., vol. 53, no. 9, pp. 598-600, Apr. 2017.

[21] G. Jo, J. Lee, J. Noh, S. Lee, and J. Lee, "Acquisition and tracking performance of satellite navigation system signal using tiered differential polyphase code," Апnи. Navigat., vol. 26, no. 1, pp. 5-11, Dec. 2019.

[22] R. Heckel and M. Soltanolkotabi, "Generalized line spectral estimation for radar and localization," in Proc. 4th Int. Workshop Compressed Sens. Theory Appl. Radar, Sonar Remote Sens. (CoSeRa), Sep. 2016, pp. 21-33.

[23] R. Heckel, V. I. Morgenshtern, and M. Soltanolkotabi, "Super-resolution radar," Inf. Inference, J. IMA, vol. 5, no. 1, pp. 22-75, 2016.

[24] R. Frank, S. Zadoff, and R. Heimiller, "Phase shift pulse codes with good periodic correlation properties (corresp.)," IEEE Trans. Inf. Theory, vol. IT-8, no. 6, pp. 381-382, Oct. 1962.

[25] D. Chu, "Polyphase codes with good periodic correlation properties (corresp.)," IEEE Trans. Inf. Theory, vol. IT-18, no. 4, pp. 531-532, Jul. 1972.

[26] B. S. Sharif, J. Neasham, O. R. Hinton, and A. E. Adams, "A computationally efficient Doppler compensation system for underwater acoustic communications," IEEE J. Ocean. Eng., vol. 25, no. 1, pp. 52-61, Jan. 2000.

[27] R. Diamant, A. Feuer, and L. Lampe, "Choosing the right signal: Doppler shift estimation for underwater acoustic signals," in Proc. 7th ACM Int. Conf. Underwater Netw. Syst. (WUWNet), 2012, p. 27.

[28] J. A. Zagzebski, Essentials of Ultrasound Physics. St. Louis, MO, USA Mosby, 1996.

[29] M. Abramowitz and I. A. Stegun, Handbook of Mathematical Functions: With Formulas, Graphs, and Mathematical Tables, vol. 55. US Government Printing Office, 1964.

[30] M. M. Saad, C. J. Bleakley, and S. Dobson, "Robust high-accuracy ultrasonic range measurement system," IEEE Trans. Instrum. Meas., vol. 60, no. 10, pp. 3334-3341, Oct. 2011.

[31] (2021). Optitrack System Overview. [Online]. Available: https:// optitrack.com/cameras/primex41/

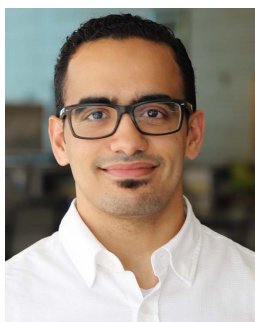

Mohammed H. AlSharif (Student Member, IEEE) received the B.S. degree (Hons.) in electrical engineering from the King Fahd University of Petroleum and Minerals, Dhahran, Saudi Arabia, in 2012, and the M.S. degree in electrical engineering from King Abdullah University of Science and Technology, Thuwal, Saudi Arabia, in 2016, where he is currently pursuing the Ph.D. degree.

He was a Visiting Scholar at California Institute of Technology, Pasadena, CA, USA, for nine months in 2020. His current research interests are in the areas of signal processing, signal design, and machine learning with applications in indoor localization, motion tracking, and hand gesture recognition.

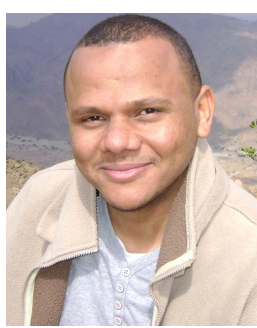

Mohamed Saad (Member, IEEE) received the Ph.D. degree from the School of Computer Science and Informatics, University College Dublin (UCD), Dublin, Ireland, in September 2013.

$\mathrm{He}$ is a Researcher at Insight Research Centre for Data Analytics, UCD. His research interest is in the areas of digital signal processing, localization the, IoT, deep learning, and machine learning.

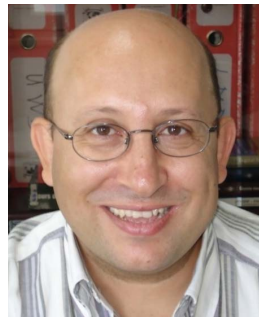

Mohamed Siala (Member, IEEE) received the general engineering degree from Ecole Polytechnique, Palaiseau, France, in 1988, and the specialization engineering degree in telecommunications and the $\mathrm{Ph} . \mathrm{D}$. degree in digital communications from Telecom ParisTech, Paris, France, in 1990 and 1995, respectively.

From 1990 to 1992, he was with Alcatel RadioTelephones, Colombes, France, working on the implementation of the GSM physical layer. In 1995 , he joined Wavecom, Issy-les-Moulineaux, France, where he worked on advanced multicarrier communications and channel estimation for low-orbit mobile satellite communications. From 1997 to 2001, he worked at Orange Labs, Issy-les-Moulineaux, on the assessment of the performance of the physical layer of 3G systems and participated actively in their standardization. In 2001, he joined SUP'COM, Tunis, Tunisia, where he is currently a Full Professor. His research interests are in the areas of digital and wireless communications with special emphasis on waveform design for advanced multicarrier systems and ARQ, channel estimation, synchronization, adaptive modulation and coding, MIMO systems, massive MIMO, spacetime coding, relaying, cooperative networks, and cognitive radio. He holds respectively 40 and 221 articles in prestigious journals and conferences and six patents.

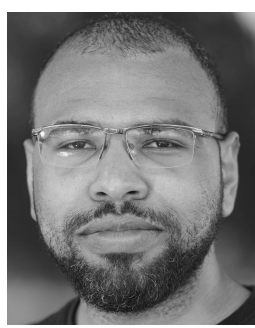

Mohanad Ahmed (Member, IEEE) received the B.Sc. degree in electrical and electronic engineering from the University of Khartoum, Khartoum, Sudan, in 2011 and the M.Sc. degree in electrical engineering from the King Fahd University of Petroleum and Minerals, Dhahran, Saudi Arabia, in 2015.

He was a Lecturer with the University of Khartoum between 2016 and 2018. His research interests include signal processing and embedded systems with focus on indoor localization. He is currently a Researcher with the King Abdullah University of Science and Technology (KAUST), Thuwal, Saudi Arabia.

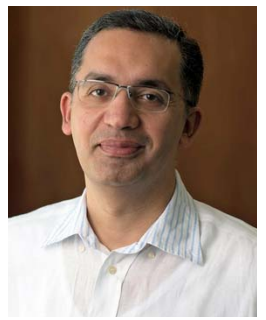

Tareq Y. Al-Naffouri (Senior Member, IEEE) received the B.S. degree (Hons.) in mathematics and electrical engineering from the King Fahd University of Petroleum and Minerals, Dhahran, Saudi Arabia, the M.S. degree in electrical engineering from the Georgia Institute of Technology, Atlanta, GA, USA, in 1998, and the Ph.D. degree in electrical engineering from Stanford University, Stanford, CA, USA, in 2004 .

He was a Visiting Scholar with the California Institute of Technology, Pasadena, CA, in 2005 and summer 2006. He was a Fulbright Scholar with the University of Southern California, Los Angeles, CA, in 2008. He is currently a Professor with the Electrical Engineering Department, King Abdullah University of Science and Technology (KAUST), Thuwal, Saudi Arabia. His research interests lie in the areas of sparse, adaptive, and statistical signal processing and their applications to wireless communications and localization, machine learning, and network information theory. He has over 300 publications in journal and conference proceedings and 20 issued/pending patents.

Dr. Al-Naffouri is the recipient of the IEEE Education Society Chapter Achievement Award in 2008 and Al-Marai Award for innovative research in communication in 2009. He was an Associate Editor of IEEE TRANSACTIONS ON SIGNAL PROCESSING 2013-2018. 